OPEN ACCESS

Edited by:

Wannan Tang,

University of Oslo, Norway

Reviewed by:

Hyungju Park

Korea Brain Research Institute,

South Korea

Frank Kirchhoff,

Saarland University, Germany

${ }^{*}$ Correspondence:

Natalina Salmaso

natalina.salmaso@carleton.ca

Specialty section:

This article was submitted to

Non-Neuronal Cells,

a section of the journal

Frontiers in Cellular Neuroscience

Received: 20 December 2020 Accepted: 26 April 2021

Published: 20 May 2021

Citation:

McNeill J, Rudyk C, Hildebrand ME and Salmaso N (2021) lon Channels and Electrophysiological Properties of Astrocytes: Implications for Emergent

Stimulation Technologies.

Front. Cell. Neurosci. 15:644126. doi: 10.3389/fncel.2021.644126

\section{Ion Channels and}

\section{Electrophysiological Properties of Astrocytes: Implications for Emergent Stimulation Technologies}

\author{
Jessica McNeill, Christopher Rudyk, Michael E. Hildebrand and Natalina Salmaso* \\ Department of Neuroscience, Carleton University, Ottawa, ON, Canada
}

Astrocytes comprise a heterogeneous cell population characterized by distinct morphologies, protein expression and function. Unlike neurons, astrocytes do not generate action potentials, however, they are electrically dynamic cells with extensive electrophysiological heterogeneity and diversity. Astrocytes are hyperpolarized cells with low membrane resistance. They are heavily involved in the modulation of $\mathrm{K}^{+}$and express an array of different voltage-dependent and voltage-independent channels to help with this ion regulation. In addition to these $\mathrm{K}^{+}$channels, astrocytes also express several different types of $\mathrm{Na}^{+}$channels; intracellular $\mathrm{Na}^{+}$signaling in astrocytes has been linked to some of their functional properties. The physiological hallmark of astrocytes is their extensive intracellular $\mathrm{Ca}^{2+}$ signaling cascades, which vary at the regional, subregional, and cellular levels. In this review article, we highlight the physiological properties of astrocytes and the implications for their function and influence of network and synaptic activity. Furthermore, we discuss the implications of these differences in the context of optogenetic and DREADD experiments and consider whether these tools represent physiologically relevant techniques for the interrogation of astrocyte function.

Keywords: glia, physiology, ion channels, calcium, potassium, sodium, optogenetics, DREADDs

\section{INTRODUCTION}

Astrocytes comprise a heterogeneous population of macroglial cells that are the most abundant neural cell type in the central nervous system (CNS). Similar to both neurons and oligodendrocytes, astrocytes arise from the neural stem cell pool (Sloan and Barres, 2014). The process of gliogenesis in rodents begins around embryonic day 16-18 with the majority of cortical astrogliogenesis likely occurring in the postnatal period where a substantial increase in glial numbers are observed during the second and third postnatal weeks (Abney et al., 1981; Qian et al., 2000; Bushong et al., 2004; Freeman, 2010). The extensive morphological and functional heterogeneity of astrocytes is in part driven by their place of birth and neuronal neighbors during the course of development (Lanjakornsiripan et al., 2018; Bayraktar et al., 2020). This may be mediated, in part, through the release of specific neurotransmitters or neurotrophic factors from these nearby neurons. At least in cortical development, neuronal heterogeneity induces differential astroglial phenotypes (Bayraktar et al., 2020). 
Morphologically, astrocytes can take many forms, though they are perhaps best known for their protoplasmic shape; a smaller soma surrounded by numerous processes that extend outwards (Sofroniew and Vinters, 2010), giving them a "starlike" shape for which they are named. In addition to these protoplasmic astrocytes, which predominantly exist in gray matter, there are fibrous astrocytes which are found throughout the white matter (Sofroniew and Vinters, 2010). The somata of these cells orient themselves in perpendicular rows between the axon bundles while their processes make connections to nodes of Ranvier (Oberheim et al., 2009). These two dominant morphological types of astrocytes are by no means an exhaustive list; velate astrocytes of the olfactory bulb and cerebellum, Bergmann glia of the cerebellum, Müller glia in the retina, pituicytes of the neurohypophysis, radial glia, and Gomori astrocytes of the hypothalamus all represent morphologicallydistinct classes of astrocytes (Verkhratsky and Nedergaard, 2018; Khakh and Deneen, 2019).

Astrocytes display even greater diversity in their functional roles. Previously believed to only provide structural support to neurons, it is now well-established that astrocytes are key regulators of CNS homeostasis. Some of these homeostatic functions include the buffering of ions like potassium $\left(\mathrm{K}^{+}\right)$, sodium $\left(\mathrm{Na}^{+}\right)$, and protons $\left(\mathrm{H}^{+}\right)$, and the regulation of neurotransmitters. Astrocytes form an integral part of the tripartite synapse; their processes encompass the synapse, allowing them to remove excess neurotransmitters from the cleft. This is particularly important for the excitatory neurotransmitter, glutamate: astrocytes are responsible for removing and breaking down almost all central extracellular glutamate (Mahmoud et al., 2019). These glutamatergic transporters are also critical for modulating neuronal plasticity; for example, downregulation of the glutamate-1 transporter has been shown to impair long-term potentiation (LTP; Li Y.-K. et al., 2012).

In addition, astrocytes contribute to CNS homeostasis by forming an integral part of the blood-brain barrier; their endfeet surround the cerebral capillaries as part of the neurovascular unit (Liedtke et al., 1996; Wilhelm et al., 2016). Thus, they play a key role in modulating the entry of molecules into the CNS; permitting access for essential substances like nutrients while preventing access of potentially harmful agents like oxidants (Wilhelm et al., 2016). Astrocytes are also crucial for regulating $\mathrm{pH}$ levels, modulating oxidative stress, and providing energy substrates to neurons. For example, astrocytes are key metabolizers of glucose, the main source of energy (ATP) production in the brain (Prebil et al., 2011).

Astrocytes are also key players in CNS injury response, undergoing morphological and functional changes in a process known as reactive astrogliosis. Their response differs according to the extent and cause of the injury and includes molecular, morphological, and physiological changes (Sofroniew and Vinters, 2010). Reactive astrocytes can produce neurotoxic or neuroprotective effects. Two distinct classifications of reactive astrocytes, termed "A1" and "A2" (neurotoxic and neuroprotective, respectively) have recently been characterized (Liddelow et al., 2017), though it is likely more phenotypes exist.
Despite the accumulating evidence demonstrating the extensive regional and sub-regional diversity of astrocytes, there remains very little understanding of how the electrophysiological properties of astrocytes may diverge across these subpopulations. A large body of evidence in the field suggests that astrocytes may not be as "electrically silent" as previously believed, so characterizing differences in these electrophysiological properties will be important for understanding the functional differences of astroglial cells. Moreover, the increased use of technologies such as designer receptors activated by designer drugs (DREADDs) and optogenetics in astrocytes, augments the need to understand the physiological properties of astrocytes as these properties will be critical for the future application of these tools to this cell population. A greater knowledge of astrocyte physiology will inform experimental design, determine the physiological relevance (or not) of specific electrical stimulation experiment(s) and help with acknowledging the limitations of each. In the remainder of this review, we will highlight the regional differences in astrocyte physiology, and discuss the implications for optogenetic and DREADD manipulation of astrocytes.

\section{HETEROGENEITY OF ASTROCYTE MEMBRANE POTENTIAL, RESISTANCE AND CURRENT PATTERNS UNDER BASAL AND REACTIVE CONDITIONS}

\section{Astrocytes Are Electrically Active Cells}

Though neurons are the main excitable cell type of the brain, astrocytes are not "electrically silent" cells. Astrocytes have a hyperpolarized membrane (Du et al., 2016) that typically rests below that of neurons (see section below; Bolton et al., 2006; Zhou et al., 2006), in contrast to the majority of non-excitable cells that have relatively depolarized membrane potentials. Though they cannot generate an action potential, astrocytes are able to respond biochemically to stimuli within their environment, especially ions and neurotransmitters. Astrocytic membranes are rich with several cation and anion channels (including both voltage-dependent and voltage-independent channels), which help with the regulation of ions such as $\mathrm{Na}^{+}, \mathrm{K}^{+}, \mathrm{Ca}^{2+}$ and $\mathrm{Cl}^{-}$, in addition to contributing to the resting membrane potential (RMP), resting conductance and intracellular signaling within astrocytes (Parpura et al., 2011; Ryoo and Park, 2016). Several of these channels have permeability properties that are independent of voltage (i.e., TWIK 1, TREK 1 -see "Voltage-Independent $\mathrm{K}^{+}$Channels Contribute to Passive Conductance" section), but many are voltage-dependent (such as delayed-rectifying potassium channels-see "Voltage-Dependent $\mathrm{K}^{+}$Channels Have Distinct Subcellular Localization" section). Therefore, astrocytes are a dynamic cell type with functional and signaling properties that vary with changes in membrane potential. In addition to ion-permeable channels, astrocytes also express several electrogenic transporters to help facilitate the exchange of ions across their membrane. For example, the $\mathrm{Na}^{+}-\mathrm{K}^{+}$-ATPase pump exchanges three $\mathrm{Na}^{+}$ions out for every two $\mathrm{K}^{+}$ions in, and the 
$\mathrm{Na}^{+}-\mathrm{K}^{+}-2 \mathrm{Cl}^{-}$-cotransporter pump exchanges $\mathrm{Na}^{+}, \mathrm{K}^{+}$, and $\mathrm{Cl}^{-}$, with an accompanying influx of water into the cell (Bellot-Saez et al., 2017). These ion channels and transporters represent an important facet of astrocyte physiology; astrocytic $\mathrm{Ca}^{2+}$ signaling represents another.

Calcium signaling in astrocytes plays an important role in facilitating the bidirectional communication between neurons and astrocytes at the synapse (for a more complete review on neuron-astrocyte interactions at the synapse, see Allen and Eroglu, 2017). Astrocytes express a plethora of ionotropic and metabotropic receptors, enabling a diverse set of responses to neurotransmitters such as glutamate, serotonin, dopamine, and GABA (Verkhratsky et al., 2019). Neurotransmitter binding to astrocytes can induce intracellular $\mathrm{Ca}^{2+}$ signals, and the magnitude, localization and time course of these signals vary significantly depending on the stimulus and synaptic network involved (Araque et al., 2014). However, this neuron to astrocyte communication is not the only form of interaction between these cell types. Astrocytes are able to modulate neuronal activity through the release of several active factors such as glutamate, ATP and D-serine in a process known as gliotransmission (Parpura et al., 1994; Cotrina et al., 2000; Henneberger et al., 2010; Araque et al., 2014; Perez et al., 2017). This process is partly mediated by intracellular $\mathrm{Ca}^{2+}$ signaling pathways (Araque et al., 2014). The release of these gliotransmitters from astrocytes is known to regulate synaptic transmission and plasticity. Changes in the frequency of miniature and spontaneous excitatory postsynaptic currents (EPSCs) and inhibitory postsynaptic currents (IPSCs), and the modulation of both LTP and longterm depression (LTD) have all been observed following the release of gliotransmitters from astrocytes across several different brain regions including the hippocampus, cortex and cerebellum (Kang et al., 1998; Brockhaus and Deitmer, 2002; Takata et al., 2011; Navarrete et al., 2012; Araque et al., 2014). Calcium signaling in astrocytes has also been shown to stimulate the $\mathrm{Na}^{+}-\mathrm{K}^{+}$-ATPase pump, leading to a decrease in extracellular $\mathrm{K}^{+}$ and subsequent neuronal hyperpolarization and suppression of baseline excitatory activity (Wang et al., 2012).

\section{Astrocytes Display a Highly Negative Resting Membrane Potential}

Compared to their neuronal counterparts, astrocytes display a more hyperpolarized, or negative, RMP (Bolton et al., 2006). While neuronal membranes typically rest at between approximately $-50 \mathrm{mV}$ and $-70 \mathrm{mV}$ (Zaitzev et al., 2012; Shen et al., 2018; Fernandez et al., 2019), the RMP of astrocytes is typically lower. However, specific astrocyte RMP values vary substantially across the CNS. For example, mature astrocytes of the CA1 region of the hippocampus have an RMP of about $-80 \mathrm{mV}$ (Tang et al., 2009; Deemyad et al., 2018), whereas astrocytes of the optic nerve have an average RMP of $-62 \mathrm{mV}$ (Butt and Jennings, 1994). A comparison between telencephalic astrocytes found that those of the stratum oriens and stratum pyramidale regions of the hippocampus had a significantly more negative (RMP of $-90 \mathrm{mV}$ ) average membrane potential compared to those of the layers V and VI of the cortex (with an RMP of about $-85 \mathrm{mV}$; Mishima and Hirase, 2010).
There is also extensive heterogeneity of astrocytes within the same region; astrocytes of the optic nerve have an RMP ranging from -25 to $-80 \mathrm{mV}$ (Bolton et al., 2006) whereas those in the ventral tegmental area (VTA) ranged from -60 to $-90 \mathrm{mV}$ (Xin et al., 2019). The hippocampus has proven to have a diverse pool of astrocytes; astrocytes in this area have RMPs ranging from $-80 \mathrm{mV}$ in the CA1 and stratum radiatum subregions (Zhong et al., 2016; Deemyad et al., 2018) to a more negative RMP $(-90 \mathrm{mV})$ in the stratum oriens and stratum pyramidale (Mishima and Hirase, 2010).

In neurons, the RMP is important for setting the threshold, propensity, and frequency of action potentials. In astrocytes, which lack action potentials, the highly negative (hyperpolarized) $\mathrm{RMP}$ is critical for enabling and regulating homeostatic functions such as $\mathrm{K}^{+}$buffering and even neurotransmitter reuptake (Zhou et al., 2006; Ryoo and Park, 2016). Variability in astrocytic RMPs throughout the CNS may therefore reflect differences in (some) astrocytic functions.

The underlying reason(s) for differences in astrocyte RMP across brain regions and subtypes have not been fully elucidated, but it is likely a result of a complex interplay between numerous extrinsic and intrinsic factors. An astrocyte's immediate environment and neuronal input may drive heterogeneity in RMP values across the CNS. The morphological characteristics of individual astrocytes may also play a role in determining RMP. For example, a heavily branched astrocyte with numerous processes and greater membrane surface area may have a higher number of ion channels, particularly leak channels (i.e., $\mathrm{K}^{+}$), that could in part explain some of the differences in astrocyte RMP (relationships between morphology, RMP heterogeneity, and differential astrocytic $\mathrm{Ca}^{2+}$ signaling-are further discussed in "Ca ${ }^{2+}$ signaling pathways in astrocytes"). However, as specific morphological classes of astrocytes have not been linked with particular ranges of RMPs, it is highly probable that several other factors also influence the RMP of an astrocyte, such as intracellular signaling pathways (see Figure 1).

One intracellular signaling cascade that may influence the RMP of astrocytes is the cAMP/PKA pathway. Bolton et al. (2006) demonstrated that incubation of astrocytes with a cAMP analog (to activate adenylate cyclase) hyperpolarized their mean RMP. The addition of a PKA inhibitor caused a significant depolarization of the astrocytic membrane, but this effect was only partially reversed with the cAMP analog, suggesting that cAMP influences astrocytic RMP via both PKA-dependent and-independent pathways (Bolton et al., 2006). Therefore, the variability of astrocytic RMP may be mediated by differences in intracellular cAMP/PKA signaling. In astrocytes, this $c A M P / P K A$ pathway has been linked to changes in cell morphology and gene expression. A recent study found that over 6,000 astroglial gene transcripts were differentially regulated by cAMP signaling; gene ontology revealed associations with pathways controlling antioxidant activity, cell metabolism, and ion transporters (Paco et al., 2016). For example, numerous ion channels, pumps and transporters such as $\mathrm{Kcn} 2$ (for $\mathrm{K}^{+}$) and ATP2a2 (for $\mathrm{Ca}^{2+}$ ) were all upregulated by cAMP (Paco et al., 2016). Given the critical role of cAMP in these various functions, 
a)

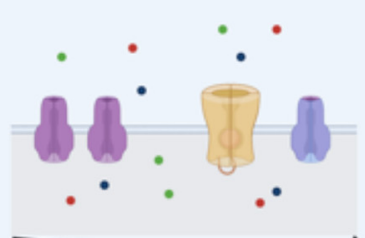

b)
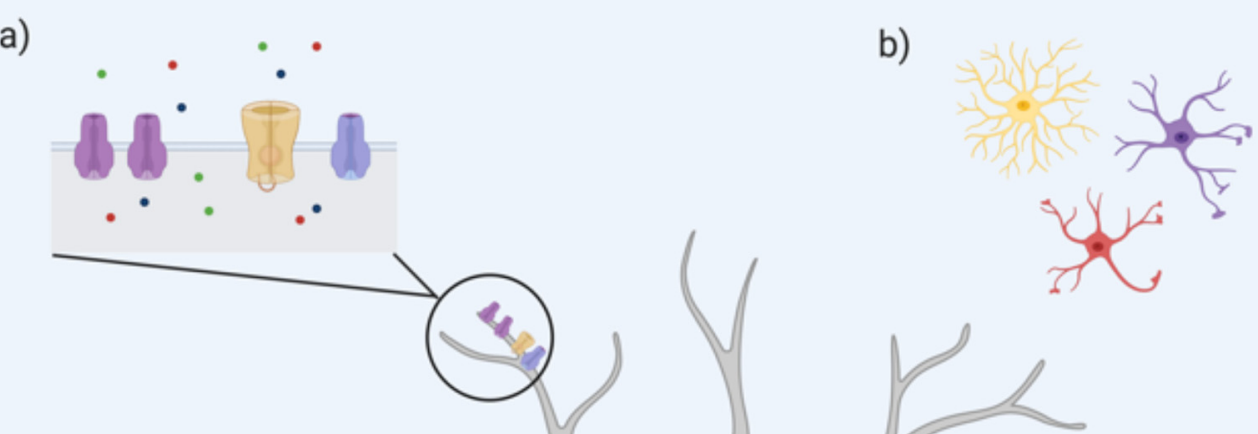

c)

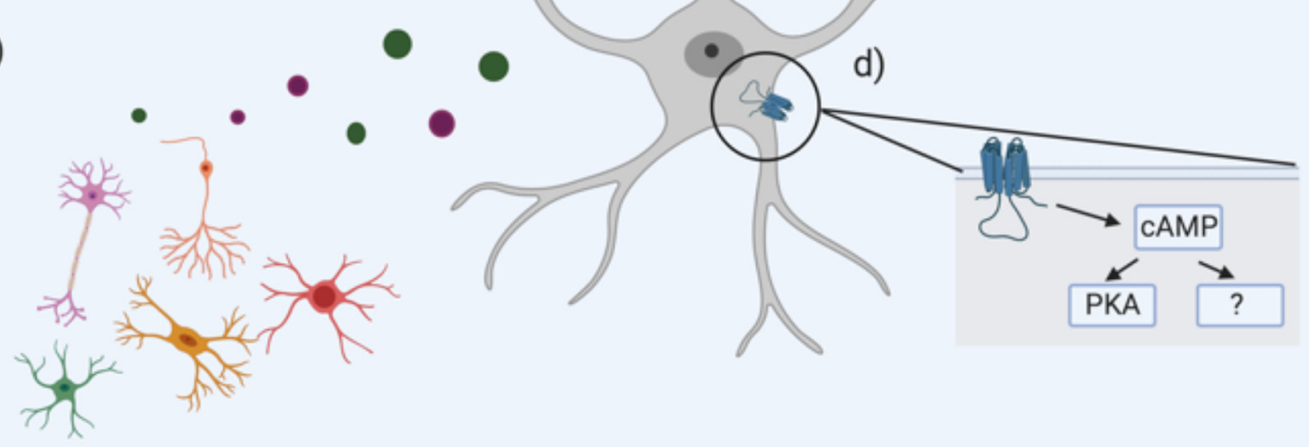

FIGURE 1 | An astrocyte's resting membrane potential (RMP) is likely influenced by multiple extrinsic and intrinsic factors including (A) ion channel subtype expression and density, particularly $\mathrm{Ca}^{2+}, \mathrm{Na}^{+}$, and $\mathrm{K}^{+}$channels (ions represented by green, red, and blue circles, respectively); (B) astrocyte morphology; (C) neighboring cells including neurons, oligodendrocytes, microglia, and other astrocytes through the release of neurotransmitters, gliotransmitters, and other factors; (D) intracellular astrocyte signaling cascades such as the cAMP pathway. CAMP PKA-dependent and PKA-independent mechanisms have been proposed to influence astrocyte RMP (Bolton et al., 2006).

the differences in astrocyte RMP may be an electrophysiological hallmark of critical differences in cAMP signaling of astrocytes between and within regions (see Figure 1).

\section{Astrocytes Are Characterized by Low Membrane Resistance}

In addition to their negative RMP state, astrocytes typically have a dramatically lower membrane input resistance than neurons under basal conditions (Zhou et al., 2006; Ma et al., 2014; Du et al., 2015; Xin et al., 2019), suggesting a relatively high overall permeability to ions at rest. The low input resistance of astrocytes makes it particularly challenging to study their biophysical properties using electrophysiological approaches (for example, the low membrane resistance can cause a large portion of the voltage drop to occur across the electrode tip rather than across the cell membrane change; Ma et al., 2014). Nonetheless, the available data suggests astrocytic membrane resistance differs across brain regions, and even within the same region. For example, VTA astrocytes have, on average, a significantly lower membrane resistance compared to those of the cortex or hippocampus (approximately $1 \mathrm{M} \Omega$ in the VTA compared to approximately $3 \mathrm{M} \Omega$ in the cortex and hippocampus; Xin et al., 2019), though no differences have been noted between cortical and hippocampal astrocytes (Mishima and Hirase, 2010; Xin et al., 2019). However, several studies have found differences in membrane resistance amongst astrocytes within the hippocampus (Isokawa and McKhann, 2005; Zhong et al., 2016), suggesting that the membrane resistance of only some hippocampal astrocytes are comparable to those in the cortex. In one study, two distinct electrophysiological phenotypes of astrocytes were identified in the stratum radiatum; one subclass was defined by a variable input resistance with an overall mean input resistance significantly higher than the other subclass (Zhong et al., 2016).

Precisely what these differences mean is not fully understood, however, because input resistance is inversely proportional to overall ion permeability across the membrane, these differences might represent variability in the capacity of astrocytes to conduct/transport ions in and out of the cell. Therefore, differences in membrane resistance could offer significant insight into the ability of astrocytes to buffer various ions as well as resultant downstream intracellular signaling pathways driven by these ions.

It is also important to consider how morphology, and specifically, membrane surface area, may influence membrane resistance. An increase in the number or length of astrocytic 
processes and therefore, membrane surface area, will lead to an increase in leak channels (if channel density is equal across these membrane processes), and thus an increase in overall membrane conductance. An increase in membrane conductance will directly result in a decrease in membrane resistance. While differences in morphology alone may be insufficient to explain the heterogeneity in astrocytic membrane resistance, it is likely that it is a contributing factor.

Challenges to the CNS have also been noted to influence membrane resistance in astrocytes, though these changes are variable and appear to depend on the type and extent of the challenge. Following a unilateral entorhinal cortex lesion, astrocytes of the denervated layer in the dentate gyrus had an increase in membrane resistance that persisted for up to 10 days post-lesion (Schröder et al., 1999). Another study observed changes of membrane resistance in some astrocytes, but not others, following incubation with high $\left[\mathrm{K}^{+}\right]$, a model of early astrocyte activation (Neprasova et al., 2007). A decrease in the membrane resistance of hippocampal astrocytes in slice was noted following exposure to ammonium (a model of hepatic encephalopathy; Stephan et al., 2012).

In a cortical freeze lesion model, changes in the membrane resistance of astrocytes were noted though these changes varied significantly depending on the relative location of the astrocytes to the injury site (Bordey et al., 2001). Increased membrane resistance was noted in layer I astrocytes of a lesioned cortex compared to controls but astrocytes in the "hyperexcitable" zone (characterized by the epileptiform activity of neurons upon stimulation) showed virtually no changes in membrane resistance (Bordey et al., 2001). Additionally, this model induced a proliferative zone; an area surrounding the lesion characterized by proliferating astrocytes (Bordey et al., 2001). Interestingly, astrocytes in this proliferative zone had a mean membrane resistance that was significantly higher than astrocytes of the hyperexcitable zone under both control and lesioned conditions (Bordey et al., 2001).

The changes in astrocytic membrane resistance following disturbances to the CNS may represent compensatory mechanisms. A low membrane resistance, such as the one typically seen in astrocytes, suggests increased ionic permeability across the astrocyte's membranes. The initial increase in input resistance following a unilateral entorhinal cortex lesion (Schröder et al., 1999) suggests a reduced ability of ion conduction across the membrane in the proliferative zone. This might represent a mechanism to help regulate ion homeostasis during times of CNS perturbations. On the contrary, this increase in membrane resistance could also represent a mechanism that perpetuates CNS damage. If an increase in membrane resistance corresponds to a reduced ability to transport ions across the membrane, this could mean a reduced ability to maintain extracellular ion homeostasis, thus causing further damage.

These injury-induced changes in astrocyte membrane resistance may correspond with the changes in astrocyte morphology that characterizes reactive astrogliosis. Following CNS injury, hypertrophy of astrocytes occurs in levels correlative to the severity of the injury (Sofroniew and Vinters, 2010). Retraction of astrocytic processes and a hypertrophied cell soma means a smaller membrane surface area (and potentially fewer leak channels), which could explain an increase in membrane resistance. As astrocytes closer to the site of injury tend to undergo greater hypertrophy, this could partially explain why the membrane resistance of astrocytes might vary across different zones of the injury site. In the case of localized CNS damage (such as a lesion or ischemic event), the location of astrocytes relative to the damage may represent a significant factor in determining whether the membrane resistance increases, decreases, or remains the same. In Bordey et al.'s (2001) study, the physiological changes differed significantly amongst the different zones of the injury, suggesting this may be an important factor influencing astrocyte physiology under reactive conditions.

Reactive astrogliosis has long been recognized as a process that induces morphological, molecular and physiological changes. Data from studies that have induced CNS damage (Schröder et al., 1999; Bordey et al., 2001; Neprasova et al., 2007; Stephan et al., 2012) demonstrate clearly that the basic electrophysiological properties of astrocytes (i.e., membrane resistance) also change in response to perturbations in the CNS, though in many contexts, these changes are not well characterized and understood. As the perspective of astrocyte heterogeneity continues to develop, under basal and reactive conditions, it will be essential for the perspective of astrocyte electrophysiology to do the same.

\section{Electrophysiological Properties of Astrocytes Across Development}

Further contributing to the complexity of astrocyte physiology is that many of the other basic electrophysiological properties of astrocytes such as their membrane potential change across development (Zhou et al., 2006; Zhong et al., 2016). One study found that neonatal astrocytes $(\mathrm{P} 1-\mathrm{P} 3)$ of the stratum radiatum had a more negative membrane potential compared to mature astrocytes $(P>21)$ of the same region (approximately $-85 \mathrm{mV}$ and $-80.9 \mathrm{mV}$, respectively; Zhong et al., 2016). These changes across development highlight the caution that must be taken when generalizing electrophysiological data across studies and across timepoints.

It is currently unknown what drives these changes in the electrophysiological properties of astrocytes throughout development. In the cortex, morphological and functional heterogeneity of astrocytes is influenced greatly by neuronal heterogeneity via the release of specific neurotransmitters, ions, and neurotrophic factors (Verkhratsky and Nedergaard, 2018; Bayraktar et al., 2020); it is likely that this is also the case for the electrophysiological features of astrocytes. It is also possible these changes reflect shifts in the expression of ion transporters. For example, the $\mathrm{K}^{+}-\mathrm{Cl}^{-}$- cotransporter (KCC2) helps modulate $\mathrm{Cl}^{-}$levels in neurons through the export of $1 \mathrm{~K}^{+}$and $1 \mathrm{Cl}^{-}$across the membrane (Annunziato et al., 2013). An increase in the neuronal expression of KCC2 early in development is believed to drive the shift of GABA from an excitatory to an inhibitory neurotransmitter (Moore et al., 2019). However, KCC2 is also expressed in astrocytes (Annunziato et al., 2013; Rurak et al., 2020), and this expression increases 
across development (Rurak et al., 2020). Perhaps the increase in KCC2 expression in astrocytes, and subsequent changes in intracellular $\mathrm{K}^{+}$and $\mathrm{Cl}^{+}$levels and reversal potentials, is what drives the changes in the electrophysiological properties such as RMP over development. Moreover, increased ion transport across the membrane via transporters such as KCC2 could also influence the input resistance of astrocytes. Developmental changes in transporter expression may represent one potential mechanism that drives changes in astrocyte electrophysiology across the lifespan.

It is clear from the literature that astrocytes differ tremendously in many of their basic electrophysiological properties such as their RMP, membrane resistance, membrane currents, and selective ion permeability. The literature also shows that these properties differ significantly at both the regional and sub-regional levels. There remains a large gap in knowledge about the relationship between the specific electrophysiology properties of astrocyte subpopulations and how these properties correspond to their morphological, biochemical, and functional properties. It is likely that future studies on astrocyte electrophysiology, will result in new methods for classifying and modulating signaling within astrocyte subtypes.

\section{Astrocyte Electrophysiology: Convergence Across Species}

The evidence presented thus far for the heterogeneity of astrocyte electrophysiology is primarily derived from studies utilizing in vitro or in vivo rodent models. However, whether the electrophysiological properties of astrocytes are conserved across species is not well characterized. Furthermore, while rodent astrocytes appear to exhibit extensive heterogeneity in their electrophysiology, it is unknown whether (or to what extent) human astrocytes show similar heterogeneity in their electrophysiological properties. However, it is well established that rodent and human astrocytes do differ in many of their characteristics (Oberheim et al., 2009; Dossi et al., 2018; Miller, 2018).

Morphological and transcriptional analyses have revealed differences between astrocytes across species. For example, several studies have demonstrated that human astrocytes tend to exhibit larger soma with a greater number of processes compared to their rodent counterparts (Oberheim et al., 2006, 2009; Zhang et al., 2016). Transcriptional differences have also been noted; one study found over 600 genes enriched in human astrocytes that were not enriched in mouse astrocytes (Zhang et al., 2016). Given this divergence between species, it is possible that human astrocytes also differ in terms of their electrophysiological properties.

Few studies have been conducted that evaluate the electrophysiological properties of human astrocytes. Early studies of human astrocytes measured comparable RMPs to those observed in rodents (Bordey and Sontheimer, 1998; O'Connor et al., 1998; Hinterkeuser et al., 2000). One of these studies noted a higher membrane capacitance in human astrocytes (Bordey and Sontheimer, 1998), which is perhaps expected given the larger surface area of human astrocytes
(Bedner et al., 2020). Interestingly though, this study did note a high input resistance (Bordey and Sontheimer, 1998). However, there is contention over whether these early studies successfully analyzed astrocytes, or whether they had actually identified NG2+ glial cells (Bedner et al., 2020). A more recent study of hippocampal astrocytes from patients with temporal lobe epilepsy revealed they exhibited a passive conductance and an RMP, membrane resistance and capacitance similar to rodent astrocytes of the same region (Bedner et al., 2015, 2020). This suggests that some electrophysiological properties of astrocytes are, in fact, conserved between rodents and humans (Bedner et al., 2020). However, there lacks sufficient studies/evidence to draw any strong conclusions.

Given the heterogeneity of astrocytes in the rodent brain, it is possible that a similar diversity in electrophysiological properties exists in human astrocytes. Studies involving direct comparisons across species will also be critical; this is particularly important because of the observed differences in species morphology, and the link between morphology and membrane capacitance. Any differences between rodent and human astrocyte electrophysiology could potentially be explained by differences in morphology. However, one study did show comparable membrane capacitance values between mouse and human astrocytes (Bedner et al., 2015, 2020), which could mean other factors are influencing human astrocyte physiology such as the density of ion channel expression. At this time, too few studies have been conducted to reach reliable conclusions as to if, and to what extent, human astrocyte electrophysiology differs from their rodent counterparts.

An additional caveat of astrocyte electrophysiology research is the extensive differences in the experimental protocol. Although in vitro (i.e., cell culture and slice) and in vivo data suggests comparable findings across experimental paradigms, few, if any, studies have directly compared electrophysiological properties of astrocytes from culture, slice and in vivo samples. Therefore, this calls for caution when interpreting and extrapolating electrophysiological data across paradigms.

\section{ASTROCYTES ARE KEY REGULATORS OF $\mathrm{K}^{+}$HOMEOSTASIS}

\section{Astroglial $\mathrm{K}^{+}$Spatial Buffering Mediated Through $\mathrm{K}_{\mathrm{ir}} 4.1$ Subtype}

The combination of a highly negative RMP and a low membrane resistance make astrocytes particularly well suited for buffering potassium $\left(\mathrm{K}^{+}\right.$; Du et al., 2015), one of their most critical homeostatic functions within the CNS. The extracellular concentration of $\mathrm{K}^{+}\left(\left[\mathrm{K}^{+}\right]_{\mathrm{o}}\right)$ rests at approximately $3.0 \mathrm{mM}$, and is critical in establishing the RMP of both neurons and astrocytes (Anderson et al., 1995; Bellot-Saez et al., 2017). Interestingly, the average RMP of astrocytes is close to the equilibrium potential of $\mathrm{K}^{+}$(Somjen, 1979; Guatteo et al., 1996), thus reflecting a high resting conductance for the ion (Somjen, 1979; Dallérac et al., 2013). 
Changes in $\left[\mathrm{K}^{+}\right]_{0}$ can be indicative of increased neuronal activity (Neprasova et al., 2007). Increases in $\left[\mathrm{K}^{+}\right]_{\mathrm{o}}$ occur following neuronal excitation whereby $\mathrm{K}^{+}$clearance from the neuron is used to co-transport $\mathrm{Na}^{+}$ions out of the cell following periods of high action potential-mediated $\mathrm{Na}^{+}$influx (Hertz and Chen, 2016). Thus, regulation and uptake of extracellular $\mathrm{K}^{+}$is essential in maintaining a homeostatic balance within the CNS. While both neurons and astrocytes are capable of regulating $\mathrm{K}^{+}$ levels, this function is typically associated with astroglial cells. Several mechanisms of $\mathrm{K}^{+}$maintenance have been identified including passive spatial buffering and uptake mediated through active transporters.

The concept of $\mathrm{K}^{+}$spatial buffering was first proposed decades ago (Walz, 1982; Verkhratsky and Nedergaard, 2018). In the proposed model, $\mathrm{K}^{+}$enters astrocytes via $\mathrm{K}^{+}$permeable membrane channels and diffuses to areas of lower $\mathrm{K}^{+}$ concentration in the glial network via gap junctions connecting the glial syncytium (Higashi et al., 2001; Verkhratsky and Nedergaard, 2018). This occurs without additional energy requirements (Orkand et al., 1966; Bellot-Saez et al., 2017). The initial uptake of extracellular $\mathrm{K}^{+}$prior to its redistribution throughout the glial syncytium is mediated through many subtypes of $\mathrm{K}^{+}$-permeable channels, including both voltagedependent (i.e., inward-rectifying and $\mathrm{Kv}$ families) and independent (i.e., two-pore domain or "leak" family, see next section) $\mathrm{K}^{+}$channels. Each of these families of channels express several subtypes including TREK 1 , TWIK 1 , and Kv 3.4 and 4.3 (this is not an exhaustive list but will be the focus in the remainder of this section). Of the variants of voltage-dependent $\mathrm{K}^{+}$channels, the inward-rectifying $\mathrm{K}^{+}$channels are a family consisting of 16 channels, subdivided into seven subfamilies (Bellot-Saez et al., 2017). The $\mathrm{K}_{\mathrm{ir}} 4.1$ subtype, a weakly inwardrectifying $\mathrm{K}^{+}$channel, is the predominant subtype expressed on astrocytes (Kofuji and Newman, 2004; Brasko et al., 2017).

The robust expression of the $\mathrm{K}_{\mathrm{ir}} 4.1$ subtype is believed to contribute to the high resting conductance of $\mathrm{K}^{+}$in astrocytes (Tang et al., 2009). However, expression of the $K_{\text {ir }} 4.1$ subtype within astrocytes is variable; the channel is expressed in the spinal cord (Olsen et al., 2006), deep cerebellar nuclei, Müller glia of the retina as well as a subset of the hippocampus, but not in all astrocytes found within the hippocampus and white matter (Poopalasundaram et al., 2000; Higashi et al., 2001; Rurak et al., 2020). In a comprehensive analysis of $K_{\mathrm{ir}} 4.1$ subtype expression in glial cells, $\mathrm{K}_{\mathrm{ir}} 4.1$ immunoreactivity was enriched in astrocytic processes wrapped around blood vessels (Hibino et al., 2004) and at synapses. Enrichment of $\mathrm{K}_{\mathrm{ir}} 4.1$ at blood vessels was also noted in human tissue (Tan et al., 2008).

The channel subtype was observed on astrocytes in several regions, including the forebrain, midbrain, and hindbrain, albeit to varying degrees (Higashi et al., 2001). The percentage of total synapses covered by $\mathrm{K}_{\mathrm{ir}}$ 4.1-positive processes varied substantially between brain regions, with over $60 \%$ of synapses covered in regions such as the entorhinal cortex, the superior and inferior colliculi and the pontine nucleus, but only $30 \%-60 \%$ of synapses covered in regions such as the anterior dorsal nucleus and lateral nuclei of the thalamus and the interpeduncular nucleus. Other regions, like the mitral cell layer of the olfactory bulb, exhibited very little $\mathrm{K}_{\mathrm{ir}} 4.1$-positive processes surrounding synapses (Higashi et al., 2001).

The expression of the $\mathrm{K}_{\mathrm{ir}} 4.1$ subtype is also variable within the cortex. Benesova et al. (2012) identified distinct subpopulations of astrocytes within the cortex that differed in their extent of swelling following oxygen-glucose deprivation. These subpopulations were (nearly) uniformly distributed across each layer of the cortex but varied significantly in gene expression of several $\mathrm{K}^{+}$-related channels, including $\mathrm{K}_{\mathrm{ir}} 4.1$. There was approximately a $1.5 \log$ difference in gene expression between the subpopulations (Benesova et al., 2012). In contrast, another study found differences in $\mathrm{K}_{\mathrm{ir}} 4.1$ immunoreactivity between layers of the cortex; there was greater $\mathrm{K}_{\mathrm{ir}} 4.1$ subtype expression in cortical layers II and III compared to layers IV-VI, though this study did not distinguish between (possible) subtypes of astrocytes (Higashi et al., 2001).

The subregional diversity of astrocytic $\mathrm{K}_{\mathrm{ir}} 4.1$ subtype expression has been noted in other regions including the olfactory bulb and the hippocampus (Higashi et al., 2001). Each layer of the olfactory bulb, for example, has varying expression of the $\mathrm{K}_{\mathrm{ir}} 4.1$ subtype subunit, with high expression in layers such as the glomerular layer, and much lower expression in layers such as the olfactory nerve and mitral cell layers (Higashi et al., 2001). A similar pattern was seen in the hippocampal layers; almost no $\mathrm{K}_{\mathrm{ir}} 4.1$ was seen in the dentate gyrus but there was astroglial expression of the channel in the CA layers (Higashi et al., 2001). Moreover, co-localization of the $\mathrm{K}_{\mathrm{ir}} 4.1$ subtype with glial fibrillary acidic protein (GFAP), an intermediate filament protein commonly used as a marker for astrocytes, showed differences between each layer of the olfactory bulb (Higashi et al., 2001), further demonstrating the molecular and physiological heterogeneity of astrocytes.

There is some evidence to indicate heterogeneous subcellular localization of $\mathrm{K}_{\mathrm{ir}} 4.1$ in astrocytes. One study of cerebellar glia found $\mathrm{K}_{\mathrm{ir}} 4.1$ expression in the radial processes of Bergmann glia in the Purkinje cell layer, whereas astrocytes of the granule cell layer expressed $\mathrm{K}_{\mathrm{ir}} 4.1$ in both the processes and somata (Brasko et al., 2017). In contrast, Muller glia of the retina preferentially express $\mathrm{K}_{\mathrm{ir}} 4.1$ in their perivascular endfeet vessels (Kofuji et al., 2002). Similarly, $K_{\text {ir }} 4.1$ is expressed in endfeet and fine processes of astrocytes within the rat optic nerve (Kalsi et al., 2004). The function of $\mathrm{K}_{\mathrm{ir}} 4.1$ may be mediated by its subcellular localization; at perivascular endfeet, the ion channel may be important for regulating $\mathrm{K}^{+}$in the blood vessels, but those localized at the processes or somata may be more important for regulating $\mathrm{K}^{+}$ levels of the astrocyte itself. Nonetheless, the heterogeneity of subcellular $\mathrm{K}_{\mathrm{ir}} 4.1$ expression across different regions further emphasizes the extensive diversity of astrocyte electrophysiology.

The variability of astrocytic $K_{\text {ir }} 4.1$ subtype expression further demonstrates the physiological heterogeneity of astrocytes. Whilst differing levels of the $\mathrm{K}_{\mathrm{ir}} 4.1$ subtype are not necessarily indicative of differing capabilities of $\mathrm{K}^{+}$regulation, it does suggest, at the very least, that astrocytes of varying brain regions utilize alternative mechanisms to regulate $\mathrm{K}^{+}$. It appears astrocytes of the same region may also exhibit alternative mechanisms for $\mathrm{K}^{+}$regulation as they also display differing 
levels of the $K_{\text {ir }} 4.1$ subtype; and since $K_{\text {ir }} 4.1$ appears to be particularly important in generating the RMP of astrocytes, it is possible that astrocyte subtypes with different membrane potentials express different levels of this $\mathrm{K}^{+}$channel. That astrocytes might differ concomitantly in distinct features (i.e., morphological, physiological, functional) demonstrates the complexity of astrocyte heterogeneity, and the importance of further understanding the physiological diversity of this unique cell population.

\section{Voltage-Independent $\mathrm{K}^{+}$Channels Contribute to Passive Conductance}

The two-pore domain, voltage-independent $\mathrm{K}^{+}$channels $\left(\mathrm{K}_{2 \mathrm{P}}\right)$ are thought to contribute substantially to the electrophysiological properties of astrocytes (Ryoo and Park, 2016; Verkhratsky and Nedergaard, 2018). This group of "leak channels" is a 15-member family of which at least three channel subtypes have been identified in astrocytes (Seifert et al., 2009; Du et al., 2016). TREK 1, TREK 2, and TWIK 1 have been observed in astrocytes of the hippocampus (Seifert et al., 2009; Du et al., 2016), cortex (Gnatenco et al., 2002), and forebrain (Cahoy et al., 2008), though it is likely that these channels are expressed in astrocytes throughout the CNS.

Despite their voltage-independence, $K_{2 P}$ channels mediate currents at a wide range of membrane potentials and are believed to contribute to the RMP of neurons and astrocytes (Ryoo and Park, 2016; Verkhratsky and Nedergaard, 2018). In the hippocampus, passive conductance (that is a linear currentvoltage relationship) in astrocytes was reduced following a pharmacological blockade and shRNA-mediated knockdown of TREK 1 and TWIK 1 (Zhou et al., 2009; Mi Hwang et al., 2014), suggesting a contributory role of these channels to the passive conductance and $\mathrm{K}^{+}$uptake observed in astrocytes (Seifert et al., 2009). However, there is some contradictory evidence to this as the genetic deletion of TWIK and/or TREK 1 did not alter passive conductance in hippocampal astrocytes (Du et al., 2016). If TWIK 1 and TREK 1 are involved in passive conductance, then differences in astrocytic conductance throughout the CNS suggests potential variability in the expression of these leak channels throughout the brain. Since the passive conductance of astrocytes is thought to be the reason for their ability to buffer $\mathrm{K}^{+}$ (Tang et al., 2009), this suggests TREK 1 and TWIK 1 might also play a contributory role in the ability of astrocytes to buffer $\mathrm{K}^{+}$. Further research is needed to determine if indeed the expression of TREK 1 and TWIK 1 influence $\mathrm{K}^{+}$buffering, and to what extent. It is also possible (and likely) these channels are important in determining other functions in astrocytes, further highlighting the need for more research.

\section{Voltage-Dependent $\mathrm{K}^{+}$Channels Have Distinct Subcellular Localization}

Beyond $\mathrm{K}_{\mathrm{ir}} 4.1$, several other types of voltage-dependent $\mathrm{K}^{+}\left(\mathrm{K}_{\mathrm{V}}\right)$ channels, with heterogeneous biophysical properties, have been identified in astrocytes (Verkhratsky and Nedergaard, 2018). As a first example, delayed rectifying $\mathrm{K}^{+}$currents have been observed in astrocytes from the spinal cord, hippocampus, cerebellum, and cortex (Bordey and Sontheimer, 2000). The delayed rectifying $\mathrm{K}^{+}$ current ion channel subtype $\left(\mathrm{K}_{\mathrm{D}}\right)$ that mediates these currents has outward rectification and a higher conductance capability at potentials more positive than $-50 \mathrm{mV}$. Transient " $\mathrm{A}^{\text {"-type }}$ currents are a second type of $\mathrm{K}^{+}$current present in astrocytes of the cerebrum, hippocampus, spinal cord, and the optic nerve (Sontheimer, 1994). The A-type channels are rapidly activating and inactivating and also require hyperpolarization to remove the tonic inactivation before they can activate (Sontheimer, 1994; Verkhratsky and Nedergaard, 2018). There are also additional subtypes of inward-rectifying $\mathrm{K}^{+}$channels beyond $\mathrm{K}_{\mathrm{ir}} 4.1$ that have been characterized in astrocytes (Bekar et al., 2005). In astrocytes, these voltage-gated $\mathrm{K}^{+}$channels are thought to play a role in modulating membrane potential; blockade of $\mathrm{K}_{\mathrm{V}}$ channels in cortical astrocytes diminished their ability to repolarize ( $\mathrm{Wu}$ et al., 2015). Blockade of these channels also reduced the influx of $\mathrm{Ca}^{2+}$, suggesting a role of these channels in the regulation of $\mathrm{Ca}^{2+}$ entry into astrocytes (Wu et al., 2015).

Various voltage-dependent $\mathrm{K}^{+}$channels are expressed in astrocytes; Kv1.1 and Kv1.6 are seen in cortical mouse astrocytes (Smart et al., 1997); Kv1.5 has been observed in the spinal cord and brains of rats, as well as in gliomas of human patients (Preussat et al., 2003). Kv1.3 is also expressed in human gliomas (Preussat et al., 2003). In addition, the subtype and subunit composition of voltage-gated $\mathrm{K}^{+}$channels differ across astrocyte subpopulations, at least in the hippocampus. One study found three families of $\mathrm{K}^{+}$channels, $\mathrm{Kv} 4, \mathrm{Kv} 3$, and $\mathrm{Kv1}$ that each contributed a different percentage of the A-type currents observed in the hippocampus (about 70, 10 and 5\%, respectively; Bekar et al., 2005). These $\mathrm{K}^{+}$channels also exhibit distinct subcellular localization in astrocytes; the Kv3.4 subtype was expressed primarily in the processes whereas the Kv4.3 was found localized to the somata (Bekar et al., 2005). The functional implications of this have not been fully elucidated, but do suggest that there may be distinct responses of the subcellular components in astrocytes to changes in voltage. Whether the differential expression of $\mathrm{Kv}$ families in hippocampal astrocytes is comparable to astrocytes of other brain regions has not yet been characterized. However, the variability in astrocytic membrane potential, which is partially modulated through voltage-gated $\mathrm{K}^{+}$ channels, suggests there is likely variability in the expression of these channels across astrocytes of other areas.

Like Kv3.4 and Kv4.3, the Kv1.3 and Kv1.6 subtypes exhibit distinct subcellular localization. In rat astrocytes, Kv1.3 is expressed on the Golgi apparatus and the Kv1.6 subtype is on the endoplasmic reticulum (Zhu et al., 2014), thus reiterating the heterogeneous nature of astrocytes and astrocyte physiology.

At least one study has demonstrated that the cAMP/PKA pathway might influence the RMP of astrocytes (Bolton et al., 2006). It is possible that the cAMP pathway regulates RMP through the modulation of voltage-gated $\mathrm{K}^{+}$channels. In fact, one study did find that treating cultured astrocytes with activators of the cAMP pathway was sufficient to modulate the expression (both up-and downregulation) of several potassium channels (Paco et al., 2016). Activation of this pathway through neuronal input, intracellular (astrocyte) signals, environmental factors, or some combination of these, might influence the temporal and spatial expression of these channels, and ultimately 
the RMP of an astrocyte. Particularly over the course of development, these neuronal inputs, signals and, environmental influences vary greatly from region to region, and this may (in part) explain the diversity of $\mathrm{Kv}$ expression and activity in astrocytes.

Potassium-dependent activity is an integral part of CNS function, and astrocytes play a key role in regulating its levels. Throughout the CNS, astrocytes express these $\mathrm{K}^{+}$channels to varying degrees, demonstrating the physiological heterogeneity and diversity of these cells. The functional outcomes of these channel profile differences have not been completely elucidated, but several studies have found a correlation between the expression of particular $\mathrm{K}^{+}$channels and cell proliferation (Bordey et al., 2001). Additionally, given that high extracellular $\mathrm{K}^{+}$has been linked to neuronal damage, the heterogeneity of astrocytic $\mathrm{K}^{+}$channels may represent regional and subregional susceptibility to $\mathrm{K}^{+}$-induced neuronal damage. This susceptibility may be particularly exacerbated in times of stress or injury; it is possible that these functional differences may only be observed under reactive conditions, or when the system has been perturbed significantly.

\section{$\mathrm{Na}^{+}$CHANNELS ARE NOT ONLY EXPRESSED IN NEURONS}

A classic dogma is that voltage-dependent sodium $\left(\mathrm{Na}^{+}\right)$ channels are only associated with excitable cells, such as neurons, because of their role in driving an action potential. Upon membrane depolarization, these voltage-dependent $\mathrm{Na}^{+}$ channels open and allow a transient inward $\mathrm{Na}^{+}$current, which marks the initiation of the action potential (Pappalardo et al., 2016). However, these voltage-dependent $\mathrm{Na}^{+}$channels have also been identified on non-excitable cells, such as Schwann cells, microglia, and astrocytes (Black and Waxman, 2013; Pappalardo et al., 2016). Though these voltage-dependent $\mathrm{Na}^{+}$channels are not responsible for action potential initiation in astrocytes, they are thought to play an important role in some astrocytic functions, particularly ion and neurotransmitter homeostasis and reactive astrogliosis (Parpura and Verkhratsky, 2012; Pappalardo et al., 2016; Verkhratsky et al., 2019). Intracellular sodium transients in hippocampal astrocytes have been observed following Schaffer collateral stimulation, suggesting sodium signaling in astrocytes occurs in response to excitatory synaptic activity (Langer and Rose, 2009). Thus, understanding the heterogenous nature of astrocytic $\mathrm{Na}^{+}$signaling and channel expression may provide critical insight into astrocyte function.

Voltage-dependent $\mathrm{Na}^{+}$channels consist of an $\alpha$-subunit, of which there are nine isoforms $\left(\mathrm{Na}_{\mathrm{v}} 1.1-\mathrm{Na}_{\mathrm{v}} 1.9\right)$ and a $\beta$ subunit of which there are only four isoforms (Pappalardo et al., 2016; Verkhratsky and Nedergaard, 2018). Astrocyte expression of these voltage-gated $\mathrm{Na}^{+}$channels has been noted in regions such as the spinal cord, cerebellum, optic nerve, cortex, and hippocampus (Sontheimer et al., 1991; Black et al., 1995; Kressin et al., 1995; Reese and Caldwell, 1999; Schaller and Caldwell, 2003; Ziemens et al., 2019; Rurak et al., 2020). Sodium signals have also been measured in astrocytes of the white matter (Moshrefi-Ravasdjani et al., 2017). In this study, $\mathrm{Na}^{+}$transients, as determined by changes in the fluorescence of the sodium indicator, SBFI, were observed following glutamate application (Moshrefi-Ravasdjani et al., 2017).

The (gene) expression of these $\mathrm{Na}_{\mathrm{v}}$ subtypes has been noted to change across development, at least in cortical astrocytes (Rurak et al., 2020). In this study, the authors combined translating ribosome affinity purification with RNA sequencing (TRAPseq) to measure gene expression changes specifically in cortical astrocytes. Across development, there was significant differential expression of several genes that encode for voltagegated $\mathrm{Na}^{+}$channels, including SCN1A (which encodes for the $\mathrm{Na}_{\mathrm{v}} 1.1$ subtype), SCN3A $\left(\mathrm{Na}_{\mathrm{v}} 1.3\right)$, SCN8A $\left(\mathrm{Na}_{\mathrm{v}} 1.6\right)$, and SCN11A $\left(\mathrm{Na}_{\mathrm{v}} 1.9\right.$; Rurak et al., 2020). Though these differentially expressed genes were not validated using alternative methods (i.e., RT-qPCR), they do suggest that the physiological properties of astrocytes (such as $\mathrm{Na}^{+}$channels and signaling) are highly dynamic across development, and extrapolating data to other studies must be done with caution.

\section{$\mathrm{Na}^{+}$Channel Subtype Expression Is Heterogenous in Astrocytes}

Several of these $\mathrm{Na}^{+}$channel subtypes have been observed in this glial population, including $\mathrm{Na}_{\mathrm{v}} 1.2, \mathrm{Na}_{\mathrm{v}} 1.3, \mathrm{Na}_{\mathrm{v}} 1.5$, and $\mathrm{Na}_{\mathrm{v}} 1.6$ (Black et al., 1995; Schaller and Caldwell, 2003; Black and Waxman, 2013; Pappalardo et al., 2016). However, there is considerable heterogeneity in $\mathrm{Na}_{\mathrm{v}}$ subtype expression in astrocytes across the CNS. For example, one study evaluated the expression of $\mathrm{Na}_{\mathrm{v}} 1.2$ and $\mathrm{Na}_{\mathrm{v}} 1.3$ in cultured astrocytes of the spinal cord and optic nerve (Black et al., 1995). Immunocytochemistry revealed higher levels of $\mathrm{Na}_{\mathrm{v}} 1.2$ and $\mathrm{Na}_{\mathrm{v}} 1.3$ in spinal cord astrocytes compared to those of the optic nerve (Black et al., 1995). Furthermore, the researchers noted varying expression of these $\mathrm{Na}^{+}$channels in morphological subtypes of astrocytes. In particular, spinal cord astrocytes classified as "stellate" had moderate levels of $\mathrm{Na}_{\mathrm{v}} 1.2$ but the "flat" astrocytes of this region expressed only low levels of the channel. Similarly, in the optic nerve, the "stellate" astrocytes expressed low levels of $\mathrm{Na}_{\mathrm{v}} 1.2$, but this expression was negligible in astrocytes classified as "flat" (Black et al., 1995). This study demonstrates the heterogeneity of some voltage-dependent $\mathrm{Na}^{+}$ channel subtypes across the CNS and further demonstrates how there is heterogenous expression within astrocytes of a given region. Data from this study suggests astrocyte morphology may be correlated to the diversity of $\mathrm{Na}_{\mathrm{v}}$ subtype expression.

Another study in the cerebellum found heterogeneity in $\mathrm{Na}_{\mathrm{v}}$ expression amongst morphological subtypes of astrocytes in this region. In the cerebellum, $\mathrm{Na}_{\mathrm{v}} 1.6$ expression has been noted in the processes of Bergmann glia, but the subtype was not identified in astrocytes of the granule cell layer (Schaller and Caldwell, 2003), suggesting again that morphological subtypes of astrocytes express different voltage-dependent $\mathrm{Na}^{+}$ channels. Interestingly, high expression of $\mathrm{Na}_{\mathrm{v}} 1.6$ was observed in cerebellar granule cells themselves (Schaller and Caldwell, 2003), implying that $\mathrm{Na}_{\mathrm{v}}$ expression in astrocytes may, in part, be driven by the expression of these channels in neighboring neurons. Like with the $\mathrm{K}^{+}$channels discussed above, it is probable that the heterogenous expression of voltage-dependent 
$\mathrm{Na}^{+}$channels in astrocytes is the result of the interplay between various intrinsic and extrinsic factors. Understanding all these different influences will help uncover the molecular and electrophysiological underpinnings of the functional differences across astrocytes.

The heterogenous expression of $\mathrm{Na}_{\mathrm{v}} 1.6$ may have important implications about an astrocyte's ability to respond appropriately to injury. Zhu et al. (2016) demonstrated that $\mathrm{Na}_{\mathrm{v}} 1.6$ expression is significantly upregulated in hippocampal astrocytes following status epilepticus in the kainic acid model of epilepsy and that this upregulation is strongly correlated to the severity of both the seizures and the reactive astrogliosis. Interestingly, the authors noted several lines of evidence demonstrating large voltage-dependent $\mathrm{Na}^{+}$currents in reactive astrocytes following seizure (de Lanerolle and Lee, 2005), suggesting that the increase in $\mathrm{Na}_{\mathrm{v}} 1.6$ in reactive astrocytes may contribute to hyperexcitability in an epileptic brain (Zhu et al., 2016). This may be caused by a $\mathrm{Ca}^{2+}$-mediated release of glutamate from astrocytes. Upregulation of $\mathrm{Na}_{\mathrm{v}} 1.6$ could drive increases in $\mathrm{Na}^{+}$influx, which, via the $\mathrm{Na}^{+} / \mathrm{Ca}^{2+}$ exchanger, could subsequently induce increases in intracellular $\mathrm{Ca}^{2+}$ levels, and $\mathrm{Ca}^{2+}$-mediated activities like the release of glutamate (Zhu et al., 2016). In an epiletic brain, this mechanism might further drive hyperexcitability and epileptogenesis. It is therefore possible that baseline differences in $\mathrm{Na}_{\mathrm{v}} 1.6$ expression in astrocytes might be indicative of the potential risk for hyperexcitability following seizure activity.

Like $\mathrm{Na}_{\mathrm{v}} 1.6$, the $\mathrm{Na}_{\mathrm{v}} 1.5$ subtype may play an important role in reactive astrogliosis. Knockdown of $\mathrm{Na}_{\mathrm{v}} 1.5 \mathrm{mRNA}$ in primary rat cortical astrocytes resulted in impaired wound closure following a scratch injury (Pappalardo et al., 2014). Furthermore, the scratch injury induced an intracellular $\mathrm{Ca}^{2+}$ response that was attenuated by the $\mathrm{Na}_{\mathrm{v} 1.5}$ knockdown (Pappalardo et al., 2014).

Changes in astrocytic $\mathrm{Na}_{\mathrm{v}} 1.5$ expression have also been observed in other models of CNS injury. In one study, conditional knockouts lacking $\mathrm{Na}_{\mathrm{v} 1.5}$ in astrocytes were generated. Compared to wildtype animals, the conditional knockouts developed more severe clinical outcomes in an EAE model of Multiple Sclerosis, though this effect was only observed in female mice (Pappalardo et al., 2018). This suggests that $\mathrm{Na}_{\mathrm{v}} 1.5$ may be important in mediating the astrocytic response to pathological conditions, though these effects may be sex-specific. Interestingly, some of the astrocytes lacking $\mathrm{Na}_{\mathrm{v}} 1.5$ appeared to have a more simple morphology compared to those of wildtype animals (Pappalardo et al., 2018).

\section{Astrocytic $\mathrm{Na}^{+}$Currents Are Linked to Specific Morphologies}

The differences in $\mathrm{Na}_{\mathrm{v}}$ subtype expression likely underlies variation in astrocytic $\mathrm{Na}^{+}$currents and associated pharmacology. Several studies have demonstrated heterogeneity of $\mathrm{Na}^{+}$currents in two morphological subclasses of astrocytes (Sontheimer and Waxman, 1992; Sontheimer et al., 1994), suggesting different (morphological) subclasses of astrocytes may express different densities of the various $\mathrm{Na}_{\mathrm{v}}$ subtypes. Fibrous astrocytes exhibit $\mathrm{Na}^{+}$currents comparable to neurons; they tend to activate at relatively depolarized potentials and inactivate rapidly. Protoplasmic astrocytes, on the other hand, tend to activate at relatively negative potentials and inactivate more slowly. Additionally, different morphological classes of astrocytes have distinct sensitivities to tetrodotoxin, a blocker of a subset of sodium channel isoforms including $\mathrm{Na}_{\mathrm{v}} 1.1-1.4$, $\mathrm{Na}_{\mathrm{v}}$ 1.6, and $\mathrm{Na}_{\mathrm{v}} 1.7$ (Sontheimer et al., 1994). In the spinal cord, the $\mathrm{Na}^{+}$currents of astrocytes characterized as "stellate" (with numerous processes) were highly sensitive to tetrodotoxin but those that had a flat or "pancake" morphology exhibited $\mathrm{Na}^{+}$ currents that were largely resistant to the drug (Sontheimer and Waxman, 1992), highlighting the extensive diversity of the astrocyte population. This suggests morphological subtypes of astrocytes may express different $\mathrm{Na}^{+}$channel subtypes, further emphasizing the heterogeneity of this cell population.

Heterogeneous astrocytic $\mathrm{Na}^{+}$signals have also been observed in those from different regions, particularly the neocortex and hippocampus (Ziemens et al., 2019). Neocortical astrocytes exhibited larger intracellular $\mathrm{Na}^{+}$transients following glutamate application in slice compared to those of the hippocampus (Ziemens et al., 2019).

The full extent to which the expression of these sodium channels and currents vary between astrocytes throughout the CNS has not been fully delineated but is imperative given the current evidence which suggests voltage-dependent $\mathrm{Na}^{+}$ channels are involved in several critical astrocytic functions such as the regulation of ion channels and in response to CNS injury such as epileptogenesis (Qiao et al., 2013; Zhu et al., 2016). It is already established that $\mathrm{Na}^{+}$currents appear to differ between morphological subtypes of astrocytes but whether these properties also differ between other subtypes of astrocytes remains to be explored. Nonetheless, the differences in $\mathrm{Na}_{\mathrm{v}}$ subtype expression and the biophysical and pharmacological properties of $\mathrm{Na}^{+}$currents further demonstrate the electrophysiological heterogeneity of astrocytes.

\section{MULTIPLE $\mathrm{Ca}^{2+}$ SIGNALING PATHWAYS IN ASTROCYTES, AN INTEGRAL PART OF ASTROCYTE PHYSIOLOGY}

Traditionally, astrocytes were believed to be passive cells whose sole function was to provide support for neuronal function. However, seminal studies in the 1990s revealed that astrocytes are capable of responding to synaptic activity through increases in intracellular calcium $\left[\mathrm{Ca}^{2+}\right]_{\mathrm{i}}$ levels (Porter and McCarthy, 1995; Pasti et al., 1997; Kang et al., 1998; Agulhon et al., 2008). These effects are also seen in cultured human astrocytes (Navarrete et al., 2013; Hashioka et al., 2014). Since then, calcium signaling cascades have been recognized as an integral part of astrocyte physiology. Importantly, under physiological conditions, both spontaneous and receptor-activated $\mathrm{Ca}^{2+}$ signals have been observed in astrocytes (Shigetomi et al., 2019).

Astrocytes express a vast array of G-protein coupled receptors (GPCRs; Shigetomi et al., 2019). In particular, $\mathrm{G}_{\mathrm{q}}$-linked GPCRs $\left(\mathrm{G}_{\mathrm{q}}\right.$ PCRs) are coupled to internal $\mathrm{Ca}^{2+}$ stores and may be the specific link between changes in $\left[\mathrm{Ca}^{2+}\right]_{i}$ in response to neurotransmitter release at the synapse (Agulhon et al., 
2008). Activation of a $G_{q} P C R s$ leads to the hydrolysis of the membrane lipid phosphatidylinositol 4,5-biphosphate $\left(\mathrm{PIP}_{2}\right)$ via the enzyme phospholipase C (PLC; Agulhon et al., 2008). This produces diacylglycerol (DAG) and inositol 1,4,5-trisphosphate $\left(\mathrm{IP}_{3}\right)$, the latter which binds to and activates $\mathrm{IP}_{3}$ receptors on the endoplasmic reticulum membrane, thereby releasing $\mathrm{Ca}^{2+}$ from intracellular stores (Agulhon et al., 2008).

There are three isoforms of the $\mathrm{IP}_{3}$ receptor $\left(\mathrm{IP}_{3} \mathrm{R}\right.$; Sherwood et al., 2017). In astrocytes, the $I_{P_{3}} R_{2}$ subtype appears to be the primary receptor subtype driving the $\mathrm{PLC} / \mathrm{IP}_{3}$ pathway (Holtzclaw et al., 2002; Sheppard et al., 2002; Petravicz et al., 2008); deletion of $\mathrm{IP}_{3} \mathrm{R}_{2}$ resulted in a lack of spontaneous and $\mathrm{G}_{\mathrm{q}} \mathrm{PCR}$-induced elevations in $\mathrm{Ca}^{2+}$ in astrocytes (Petravicz et al., 2008). However, some studies have demonstrated $\mathrm{Ca}^{2+}$ signals still occur in the astrocytes of mice lacking the $\mathrm{IP}_{3} \mathrm{R}_{2}$ subtype (Stobart et al., 2018b), suggesting other mechanisms contribute to intracellular $\mathrm{Ca}^{2+}$ astrocytic signaling. In fact, a recent study found that the first and third isoforms of $\mathrm{IP}_{3} \mathrm{R}$ may also contribute to $\mathrm{Ca}^{2+}$ signaling in astrocytes, albeit to a lesser extent (Sherwood et al., 2017).

Nonetheless, the $\mathrm{IP}_{3} \mathrm{R}_{2}$ subtype plays a particularly important role in $\mathrm{Ca}^{2+}$ signaling. A study from Holtzclaw et al. (2002) demonstrated distinct regional astrocytic subcellular expression of $\mathrm{IP}_{3} \mathrm{R}_{2}$. Astrocytes of the hippocampus expressed $\mathrm{IP}_{3} \mathrm{R}_{2}$ in their somata and processes, though there appeared to be a higher density in the large and fine processes. This was also true for cerebellar Bergmann glia in the molecular layer, which had punctate expression of $\mathrm{IP}_{3} \mathrm{R}_{2}$ in their finer processes. In contrast, Bergmann glia of the Purkinje cell layer expressed $\mathrm{IP}_{3} \mathrm{R}_{2}$ primarily in their somata (Holtzclaw et al., 2002). The study also showed that $\mathrm{IP}_{3} \mathrm{R}_{2}$ is particularly enriched in astrocytic processes that encircle synapses, highlighting an important putative role of $\mathrm{Ca}^{2+}$ signaling in mediating the link between neurotransmission and astroglia.

\section{Spontaneous $\mathrm{Ca}^{2+}$ Oscillations in Astrocytes}

Spontaneous $\mathrm{Ca}^{2+}$ signaling events occur in the absence of neuronal activity (Parri et al., 2001) and have been observed in astrocytes throughout the CNS including in the hippocampus (Nett et al., 2002; Rungta et al., 2016), cortex, striatum, and thalamus (Parri et al., 2001; Aguado et al., 2002; Jiang et al., 2014); the presence of spontaneous $\mathrm{Ca}^{2+}$ signals have also been confirmed, at least in vitro, in human astrocytes (Navarrete et al., 2013). They have been observed in Bergmann glia of the cerebellum (Aguado et al., 2002) as well as in astrocytes of the olfactory bulb (Otsu et al., 2015). Both extracellular and intracellular $\mathrm{Ca}^{2+}$ levels are critical for driving these spontaneous oscillations (Aguado et al., 2002); removal of extracellular $\mathrm{Ca}^{2+}$ is sufficient to prevent spontaneous $\mathrm{Ca}^{2+}$ signals in vitro (Aguado et al., 2002) and the loss of the $\mathrm{IP}_{3} \mathrm{R}_{2}$ reduces spontaneous events in vivo (Petravicz et al., 2008; Jiang et al., 2016; Yu et al., 2018). However, some reports have noted spontaneous $\mathrm{Ca}^{2+}$ events even in the astrocytes of $\mathrm{IP}_{3} \mathrm{R}_{2}{ }^{-/-}$mice (Sherwood et al., 2017), suggesting that these mechanisms are not the only ones that drive spontaneous $\mathrm{Ca}^{2+}$ signals in astrocytes. Recent evidence from $\mathrm{Wu}$ et al. (2019) suggests that the morphology of astrocytes may be an important factor in driving these spontaneous $\mathrm{Ca}^{2+}$ signals- these events were more frequent in thin processes with a high surface-to-volume ratio. Given the extensive morphological heterogeneity of astrocytes, it is possible that the localization of transient $\mathrm{Ca}^{2+}$ events to thinner processes can explain some of the diversity in astroglial calcium signaling, particularly spontaneous events. The spontaneity of these $\mathrm{Ca}^{2+}$ signals fluctuate between astrocytes across the CNS, perhaps reflecting diversity in some of these processes. For example, hippocampal astrocytes, specifically of the CA1 stratum radiatum region, have a higher frequency of spontaneous $\mathrm{Ca}^{2+}$ events than those of the dorsolateral striatum (Chai et al., 2017), but fewer oscillations than those of the cortex (Navarrete et al., 2013), perhaps reflecting regional differences in the mechanisms that drive these spontaneous events.

In the hippocampus, a subset of astrocytes displayed no spontaneous $\mathrm{Ca}^{2+}$ signals, but of the astrocytes that did, there was significant variability in the frequency of these events (Nett et al., 2002). Some had fairly frequent $\mathrm{Ca}^{2+}$ oscillations at intervals between 0.5 and $2 \mathrm{~min}$, whilst others had much more irregular intervals between oscillations, with intervals that exceeded 2 mins (Nett et al., 2002). Similar heterogeneity has been observed in the thalamus; the majority of thalamic astrocytes showed multiple spontaneous $\mathrm{Ca}^{2+}$ events over a 10 -min period but several displayed only one spontaneous $\mathrm{Ca}^{2+}$ signal in the same time (Parri et al., 2001). In the somatosensory cortex, astrocytes from layer I had nearly double the frequency of spontaneous $\mathrm{Ca}^{2+}$ activity compared to those from layer II/III (Takata and Hirase, 2008).

Precisely what the differences in spontaneous $\mathrm{Ca}^{2+}$ signaling across astrocyte subpopulations mean has yet to be fully elucidated. One study found that hippocampal astrocytes which did not exhibit spontaneous $\mathrm{Ca}^{2+}$ signals did not differ in morphology or electrophysiology from those that did (Nett et al., 2002), demonstrating that the classification of astrocytes is not so straightforward as one morphological class displaying one set of electrophysiological properties, or molecular markers. Regardless, it is plausible that the differences in these spontaneous $\mathrm{Ca}^{2+}$ oscillations represent important distinctions between the various types of astrocytes. Astrocytes displaying no spontaneous $\mathrm{Ca}^{2+}$ are still able to respond to some neurotransmitter release (Nett et al., 2002), but the lack of spontaneous $\mathrm{Ca}^{2+}$ signals might indicate a decreased ability or less sensitive response to these types of events which normally evoke a $\mathrm{Ca}^{2+}$ response.

The mobilization of both intracellular and extracellular $\mathrm{Ca}^{2+}$ may be another important mechanism driving spontaneous $\mathrm{Ca}^{2+}$ events (Aguado et al., 2002). Removal of extracellular $\mathrm{Ca}^{2+}$ blocked spontaneous $\mathrm{Ca}^{2+}$ events in hippocampal astrocytes in slices (Aguado et al., 2002). Likewise, when thapsigargin, a drug which inhibits endoplasmic reticulum $\mathrm{Ca}^{2+}$-ATPase activity and thus depletes $\mathrm{ER} \mathrm{Ca}^{2+}$ stores, was applied to hippocampal slices, spontaneous $\mathrm{Ca}^{2+}$ activity in astrocytes was blocked. This suggests that intracellular $\mathrm{Ca}^{2+}$ stores are also necessary for spontaneous $\mathrm{Ca}^{2+}$ events in astrocytes (Aguado et al., 2002). The lack of spontaneous $\mathrm{Ca}^{2+}$ activity in astrocytes might therefore reflect a depletion of extracellular or intracellular 
$\mathrm{Ca}^{2+}$ signaling capacity, highlighting the influence of both extrinsic and intrinsic factors in the development of astroglial spontaneous $\mathrm{Ca}^{2+}$ activity. As with the frequency, the amplitude of these spontaneous astrocyte $\mathrm{Ca}^{2+}$ transients are variable- both inter-and intra-regionally (Aguado et al., 2002). This is true in several regions including the cortex, hippocampus, thalamus, hypothalamus, and spinal cord (Aguado et al., 2002). The heterogeneity of these spontaneous events is likely the result of regional and subregional diversity in the presence of extracellular $\mathrm{Ca}^{2+}$ and the available intracellular $\mathrm{Ca}^{2+}$ stores in astrocytes.

It is clear that there are subtle differences between astrocytes displaying spontaneous $\mathrm{Ca}^{2+}$ oscillations and those that do not (Nett et al., 2002). Furthermore, it is clear that of the astrocytes that do exhibit spontaneous $\mathrm{Ca}^{2+}$ oscillations, they differ significantly in several of their properties including the frequency and the amplitude of these signals. It is possible these distinct spontaneous $\mathrm{Ca}^{2+}$ oscillations have extensive functional consequences, making the understanding of this heterogeneity pertinent to comprehending the role of astrocytes within the CNS.

\section{Astrocytic $\mathrm{Ca}^{2+}$ Signals Differ in Subcellular Compartments}

Initial studies into astrocytic $\mathrm{Ca}^{2+}$ signaling focused on cytosolic $\mathrm{Ca}^{2+}$ signals, primarily in the somata of astrocytes, but the use of genetic encoded calcium indicators (GECIs) have enabled higher resolution visualization of localized $\mathrm{Ca}^{2+}$ transients in the processes and finer processes of astrocytes (Shigetomi et al., 2013; Stobart et al., 2018a; for a comparison of calcium imaging techniques, see Smith et al., 2018). For example, spontaneous $\mathrm{Ca}^{2+}$ transients have been observed in the somata, processes, and fine processes of striatal astrocytes (Jiang et al., 2014). These cytosolic and local transients have also been observed in astrocytes of the cortex (Agarwal et al., 2017), hippocampus (Zur Nieden and Deitmer, 2006; Jiang et al., 2014), somatosensory cortex (Wang et al., 2006), and olfactory bulb (Otsu et al., 2015).

Like the extensive inter-and intraregional variability in the frequency and amplitude of astrocytic $\mathrm{Ca}^{2+}$ signals, there is substantial data suggesting that these events are also variable across the distinct subcellular compartments of astrocytes. In particular, it seems that spontaneous $\mathrm{Ca}^{2+}$ events occur more frequently in the processes compared to the somata of astrocytes. One study found the majority of $\mathrm{Ca}^{2+}$ events $(\sim 85 \%)$ were localized to the processes, with a much smaller percentage $(\sim 10 \%)$ in the endfeet, and an even smaller percentage ( $\sim 5 \%)$ in the somata (Bindocci et al., 2017). A very similar distribution of spontaneous $\mathrm{Ca}^{2+}$ events was seen in astrocytes within the somatosensory cortex, with approximately $80 \%$ of those signals occurring in the fine processes (Kanemaru et al., 2014). In astrocytes of the stratum lucidum region of the hippocampus, spontaneous $\mathrm{Ca}^{2+}$ signals were virtually absent in the somata but were frequently observed in the processes (in fact, there was about an 8-fold greater increase in these events in the processes compared to the somata; Haustein et al., 2014). The high frequency of spontaneous $\mathrm{Ca}^{2+}$ transients in the fine processes of astrocytes relative to their somata has also been observed in the visual cortex (Asada et al., 2015).
The spontaneous $\mathrm{Ca}^{2+}$ transients observed throughout the subcellular compartments of astrocytes also differ in their ability to spread from the source event. In the CA1 region, for example, researchers identified distinct $\mathrm{Ca}^{2+}$ fluctuations; one fluctuation produced a wave-like property, spreading to adjacent areas, whereas the other produced a restricted response which the authors referred to as a microdomain (Srinivasan et al., 2015). Both types of fluctuations were displayed in the processes, and each differed from fluctuations measured from the somata (Srinivasan et al., 2015).

Spontaneous $\mathrm{Ca}^{2}$ transients, while common in astrocytes, vary tremendously in the frequency, amplitude, and even type of fluctuation they produce. These properties differ at the regional, subregional, and subcellular levels. The current literature highlights the complexity of astroglial $\mathrm{Ca}^{2+}$ signaling at the regional, sub-regional, and cellular level.

This subcellular heterogeneity of $\mathrm{Ca}^{2+}$ signals, combined with the varied expression of voltage-dependent and voltageindependent ion channels (such as those discussed previously), suggests that astrocytes possess different electrophysiological profiles amongst distinct microdomains of the cell. Thus, permitting a localized response to environmental changes. These differences in subcellular compartments may also explain voltage-dependent ion channels in astrocytes; while the relatively low membrane resistance and hyperpolarized phenotype would (generally) require highly depolarizing events to activate these channels, the intracellular heterogeneity means there could be local variability in the membrane resistance and RMP of the processes, fine processes, and soma of the astrocyte.

\section{ASTROCYTE PHYSIOLOGY INFLUENCES SYNAPTIC NETWORKS}

Thus far, we have summarized the available data on the heterogeneity of astrocyte physiology. But what do these differences mean for the greater network? Does this heterogeneity translate to functional differences between other cells in the network such as neurons? If, and how, these differences may influence the network is not fully understood, but there is some evidence to suggest they can.

$\mathrm{K}^{+}$homeostasis is a critical function of astroglia, but they are not the sole cell type that can buffer this ion. Neurons, too, are able to buffer against $\mathrm{K}^{+}$. Not all astrocytes may regulate $\mathrm{K}^{+}$equally; for example, the expression of the $\mathrm{K}_{\mathrm{ir}} 4.1$, a key channel in buffering $\mathrm{K}^{+}$, is highly variable amongst astrocytes. In areas where the $\mathrm{K}^{+}$buffering capacity of astrocytes is low, then neurons may need to be more active at regulating $\mathrm{K}^{+}$to help offset that deficit. This increased activity of neuronal transporters could drive higher energy and metabolic demands, which might, in turn, make these neurons more susceptible to electrical and chemical perturbations. The implications of this heterogeneity in $\mathrm{K}^{+}$buffering are not yet understood, but moving forward may be particularly important when considering why some neuronal populations are more susceptible to perturbations and damage than others.

Several studies have shown a "wave-like" phenomena between astrocytes; that is, a $\mathrm{Ca}^{2+}$ signal appears in one astrocyte followed 
quickly by a $\mathrm{Ca}^{2+}$ signal in another nearby (Verkhratsky, 2006). This is mediated through the extensive gap junction coupling of astrocytes, forming the "net-like" structure of the glial syncytium and demonstrates how astrocytes can influence surrounding astrocytes. Of course, $\mathrm{Ca}^{2+}$ signals are only one way astrocytes can influence each other; ions (such as $\mathrm{K}^{+}$- see "Astroglial $\mathrm{K}^{+}$ Spatial Buffering Mediated Through $\mathrm{K}_{\mathrm{ir}} 4.1$ Subtype" section) can also cross gap junctions as can energy substrates like glucose (Rouach et al., 2008) and lactate (Murphy-Royal et al., 2020).

It has also been theorized that astrocytic $\mathrm{Ca}^{2+}$ signals can influence synaptic activity. One study found that spontaneous $\mathrm{Ca}^{2+}$ signals in hippocampal astrocytes were localized to specific regions in their processes (Nett et al., 2002). These "microdomains" were typically asynchronous, leading the authors to believe the astrocytes might be influencing neurons in a synapse-specific manner. However, they did note some "wavelike" activity between processes of the same astrocyte, suggesting a potential larger influence on the greater synaptic field (Nett et al., 2002). A recent study did find a more direct effect of the glial syncytium on synaptic activity (Murphy-Royal et al., 2020). In this experiment, an acute stressor was sufficient to reduce gap junction coupling between astrocytes, leading to a decrease in energy substrate transport between astrocytes and subsequent impairment in LTP (Murphy-Royal et al., 2020), clearly demonstrating that an intact glia syncytium is necessary for synaptic plasticity.

\section{OPTOGENETICS AND DREADDS: PHYSIOLOGICALLY RELEVANT FOR THE STUDY OF ASTROCYTES?}

Understanding the physiology of astrocytes has been challenging partly because of a lack of technology sensitive enough to measure many of these properties. This has been compounded by the inability to directly target and manipulate select populations of astrocytes in vivo. In the past couple of decades, the advent of novel technologies has enabled specific cell populations, such as astrocytes, to be selectively targeted and manipulated, thus permitting more in-depth analysis of their function within the CNS. In particular, optogenetics and DREADDs have been frontrunners for targeting astrocytes.

With optogenetics, cells of interest are targeted with light-sensitive proteins, known as opsins, which generally exist as ion channels and pumps (Bang et al., 2016). The absorption of a specific wavelength by the opsin induces a conformational change, driving electrical changes across the plasma membrane (Bang et al., 2016). These cellular changes differ extensively depending on the type and variant of opsin used. Channelrhodopsin 2 (ChR2), for example, is a cation channel that is activated specifically by $473 \mathrm{~nm}$ (blue) light (Nagel et al., 2003), leading to an influx of cations (namely protons and $\mathrm{Na}^{+}$) that produces membrane depolarization (Boyden et al., 2005). In astrocytes, the cation influx (particularly of $\mathrm{Na}^{+}$) may be important for modulating neuron homeostasis and GABAergic transmission (Parpura and Verkhratsky, 2012). $\mathrm{Na}^{+}$signaling in astrocytes (particularly $\mathrm{Na}^{+}$influx mediated by voltage-dependent $\mathrm{Na}^{+}$channels) is thought to be necessary for the activity of the $\mathrm{Na}^{+} / \mathrm{K}^{+}$ATPase pump (Verkhratsky et al., 2019), suggesting a role in the maintenance of ion homeostasis. Additionally, intracellular $\mathrm{Na}^{+}$is important in controlling GABA uptake via the GABA transporter (GAT) pathways; decreases in $\mathrm{Na}^{+}$can reverse GAT-dependent transport causing GABA to be released (Verkhratsky et al., 2019). However, ChR2 can also allow the influx of $\mathrm{Ca}^{2+}$, which has been shown to cause the release of gliostransmitters like ATP (Gourine et al., 2010; Chen et al., 2013) and glutamate (Haydon and Carmignoto, 2006). ChR2 stimulation in astrocytes has been reliably shown to induce changes in intracellular $\mathrm{Ca}^{2+}$ (Li D. et al., 2012; Figueiredo et al., 2014; Perea et al., 2014; Mederos et al., 2019; Balachandar et al., 2020); these changes have been observed to enhance both excitatory and inhibitory synaptic transmission in the primary visual cortex (Perea et al., 2014). The use of ChR2 to activate astrocytes has also been shown to induce the release of ATP (Gourine et al., 2010; Figueiredo et al., 2014).

Halorhodopsin, on the other hand, is optimally activated by approximately $590 \mathrm{~nm}$ (yellow) light and drives hyperpolarization of cells through the influx of $\mathrm{Cl}^{-}$ions (Bang et al., 2016). $\mathrm{Cl}^{-}$has been shown to affect outward $\mathrm{K}^{+}$currents in cultured astrocytes (Bekar and Walz, 1999), showing that the ion has an important role in mediating astrocyte physiology. Intracellular $\mathrm{Cl}^{-}$in astrocytes has also been theorized to play a role in mediating crosstalk between neurons and astrocytes because of its impact on several astrocytic transporters, including the excitatory amino acid transporters EAAT1 and EAAT 2 (GLAST and GLT-1 in rodents, respectively), GAT 1 and 3, and NKCC1 (Wilson and Mongin, 2019). Changes in astrocyte intracellular $\mathrm{Cl}^{-}$levels are associated with EAAT activity and the movement of $\mathrm{Cl}^{-}$across the membrane is necessary for GAT function (Wilson and Mongin, 2019). Given the apparent role of $\mathrm{Cl}^{-}$signaling in astrocyte physiology, the manipulation of this ion may be important for deepening our understanding of $\mathrm{Cl}^{-}$function within an astrocyte. Moving forward, the use of halorhodopsin in astrocytes will likely become an invaluable tool. As with neurons, the functional consequences of the use of different opsins will vary significantly in astrocytes.

Like halorhodopsin, archaerhodopsin (Arch) and archaerhodopsin-T (Arch-T) also respond to yellow or green light (around $532 \mathrm{~nm}$ ) and drive hyperpolarization of a cell (El-Gaby et al., 2016). However, unlike halorhodopsin, Arch and Arch-T induce hyperpolarization through the efflux of protons (El-Gaby et al., 2016; Poskanzer and Yuste, 2016). This hyperpolarization is sufficient to induce changes in intracellular $\mathrm{Ca}^{2+}$ in astrocytes; work from Poskanzer and Yuste (2016) showed that Arch activation in cortical astrocytes evoked $\mathrm{Ca}^{2+}$ transients. These transients appeared primarily in the branches and lasted approximately the same length as those generated from spontaneous events in non-stimulated controls (Poskanzer and Yuste, 2016). However, a slight decrease in the amplitude of these $\mathrm{Ca}^{2+}$ transients was noted in the Arch-stimulated compared to the non-stimulated astrocytes (Poskanzer and Yuste, 2016), suggesting that optogenetic stimulation may recapitulate some, but not all, physiological properties of astrocytes. Which of these physiological properties are conserved may depend on 
the type of opsin used, the region of interest, and the original physiological state of the astrocyte.

Melanopsin responds to shorter wavelengths of light and is optimally activated by light of about $420-450 \mathrm{~nm}$ (blue) light (Newman et al., 2003; Wang et al., 2019). It is slightly different from other opsins as it binds to a GPCR, leading to $\mathrm{Ca}^{2+}$ signaling via activation of the $\mathrm{PLC} / \mathrm{IP}_{3}$ pathway (Mederos et al., 2019). As such, it has been suggested as a good tool for investigating the physiological effects of astrocytes. One recent study transfected melanopsin into hippocampal astrocytes and found that stimulation resulted in robust $\mathrm{IP}_{3}$-dependent $\mathrm{Ca}^{2+}$ signals in their fine processes and the release of ATP/adenosine at the synapse (Mederos et al., 2019).

Adaptations to these opsins (particularly ChR2) have been generated in recent years, including ChETA and step function opsins (SFO; Bang et al., 2016). In neurons, ChETA has been shown to induce ultrafast spiking and SFOs are known to produce prolonged, sub-threshold membrane depolarizations (Bang et al., 2016) but how these differences might translate to astrocyte activity is not well characterized.

Optogenetics provides precise spatial and temporal resolution for the manipulation of cells within the CNS. The high spatial resolution can be obtained via the viral (i.e., adeno-associated virus or lentivirus) transfection of the genetically engineered opsin to a targeted region of interest. Combined with a celltype-specific promoter, a high degree of cell specificity is attainable. The ability to precisely modulate the frequency of light pulses delivered to these regions of interest allows extensive manipulation of these cells, and thus provides a high degree of temporal resolution that is not seen in many other technologies. Optogenetics has been particularly valuable for studying neuronal function throughout the CNS, and in more recent years, the technology is being applied to astrocytes. Since the effects of depolarization and hyperpolarization on astrocyte signaling are not as well understood as these effects in neurons, the relevance and generalizability of optogenetic approaches to manipulate and understand astrocytes remains to be fully elucidated.

The use of designer receptors exclusively activated by designer drugs (DREADDs) provides an alternative to target cells, particularly astrocytes. DREADDs represent one of the most commonly employed forms of chemogenetics, in which genetically engineered receptors selectively bind ligands to drive transient changes (usually activation or inactivation) in the region of interest (Roth, 2016; Smith et al., 2016). The genetically engineered GPCRs have little to no response to endogenous ligands but a strong response to synthetic ones that are otherwise biologically inert (Bang et al., 2016). When the ligand (or actuator) is introduced, it binds to and activates the receptor, activating the downstream $\mathrm{Gq}, \mathrm{Gi}$, or Gs pathways that are coupled with these receptors.

Activation of neuronal and non-neuronal cells is mediated through DREADDs targeting the Gq signaling pathway, of which the hM3Dq is most frequently used (Alexander et al., 2009; Roth, 2016). Inhibitory DREADDs, which silence neuronal activity, target the Gi pathway, the most common being the hM4Di receptor (Roth, 2016). Traditionally, clozapine-N-oxide
(CNO) has been used as the chemical actuator for both hM3Dq and hM4Di (as well as several other DREADDs), but new evidence suggesting it can reverse-metabolize to its parent compound, clozapine (Manvich et al., 2018; Walker and Kullmann, 2020), and that it may have poor penetrance into the brain (Bonaventura et al., 2019) has led to the use of newer classes of chemical actuators including Compound 13, Compound 21, JHU37152, and JHU37160 (Bonaventura et al., 2019).

Like opsins, these receptors can be placed under the control of cell-specific promoters, restricting expression to a particular group of cells. The use of a fluorescent tag such as green fluorescent protein (GFP) allows for easy visualization of the cells expressing the opsin or DREADD of interest.

Since DREADDs use intracellular $\mathrm{Ca}^{2+}$ signaling to modulate cell activity, many have suggested they are particularly useful for studying astroglial activity given the integral role of $\mathrm{Ca}^{2+}$ signaling in astroglial function and communication; that is, the manipulation of astrocytes via the GPCR activation might be physiologically relevant. However, neither DREADDs nor optogenetics may be physiologically relevant for the study of astrocytes unless the unique physiological features of astrocytic subtypes are addressed in the experimental design.

Due to their low membrane resistance, astrocytes will likely elicit smaller current changes to optogenetic stimulation than neurons. As such, "stronger" or more "intense" stimulation may be needed to elicit a response in astrocytes. Fine-tuning of experimental guidelines is needed to determine what parameters would elicit a physiological response in astrocytes.

Another important consideration for the manipulation of $\left[\mathrm{Ca}^{2+}\right]_{\mathrm{i}}$ in astrocytes is that the mechanism which induces a rise in $\left[\mathrm{Ca}^{2+}\right]_{i}$ can influence the speed of the change in these levels (Bazargani and Attwell, 2016). This suggests that the use of opsin variants (such as channelrhodopsin 2, ChR2, and its variant ChETA), which have similar, but differing mechanisms (ChETA has faster kinetics and in neurons has been proven to have more rapid repolarization than $\mathrm{ChR} 2$; Gunaydin et al., 2010), may induce changes in $\left[\mathrm{Ca}^{2+}\right]_{\mathrm{i}}$ at varying speeds, possibly contributing to alternative downstream effects. This is further compounded by the regional and subregional diversity of astrocyte physiology previously discussed in this review, rendering rigorous pilot studies essential prior to the use of DREADDs or optogenetics to target astrocytes (see Figure 2).

One study compared several ChR2 variants; all four variants evaluated were sufficient to induce large increases in $\left[\mathrm{Ca}^{2+}\right]_{i}$ (Figueiredo et al., 2014). There were no significant differences in the overall $\left[\mathrm{Ca}^{2+}\right]_{i}$ responses or in the dynamics of these responses between the ChR2 variants (Figueiredo et al., 2014). However, another study found the $\mathrm{Ca}^{2+}$-translocating channelrhodopsin (CatCh) variant was more efficient than ChR2 in controlling $\mathrm{Ca}^{2+}$ elevations in astrocytes (Li D. et al., 2012). This implies that generalizing results from studies that employ different opsin variants may be difficult. Selecting the appropriate opsin variant to employ (i.e., to produce a particular/desired effect) will be further complicated by the diverse nature of astrocytic $\mathrm{Ca}^{2+}$ signaling.

Differences between opsin types rather than just opsin variants may also induce significantly different effects. A recent 


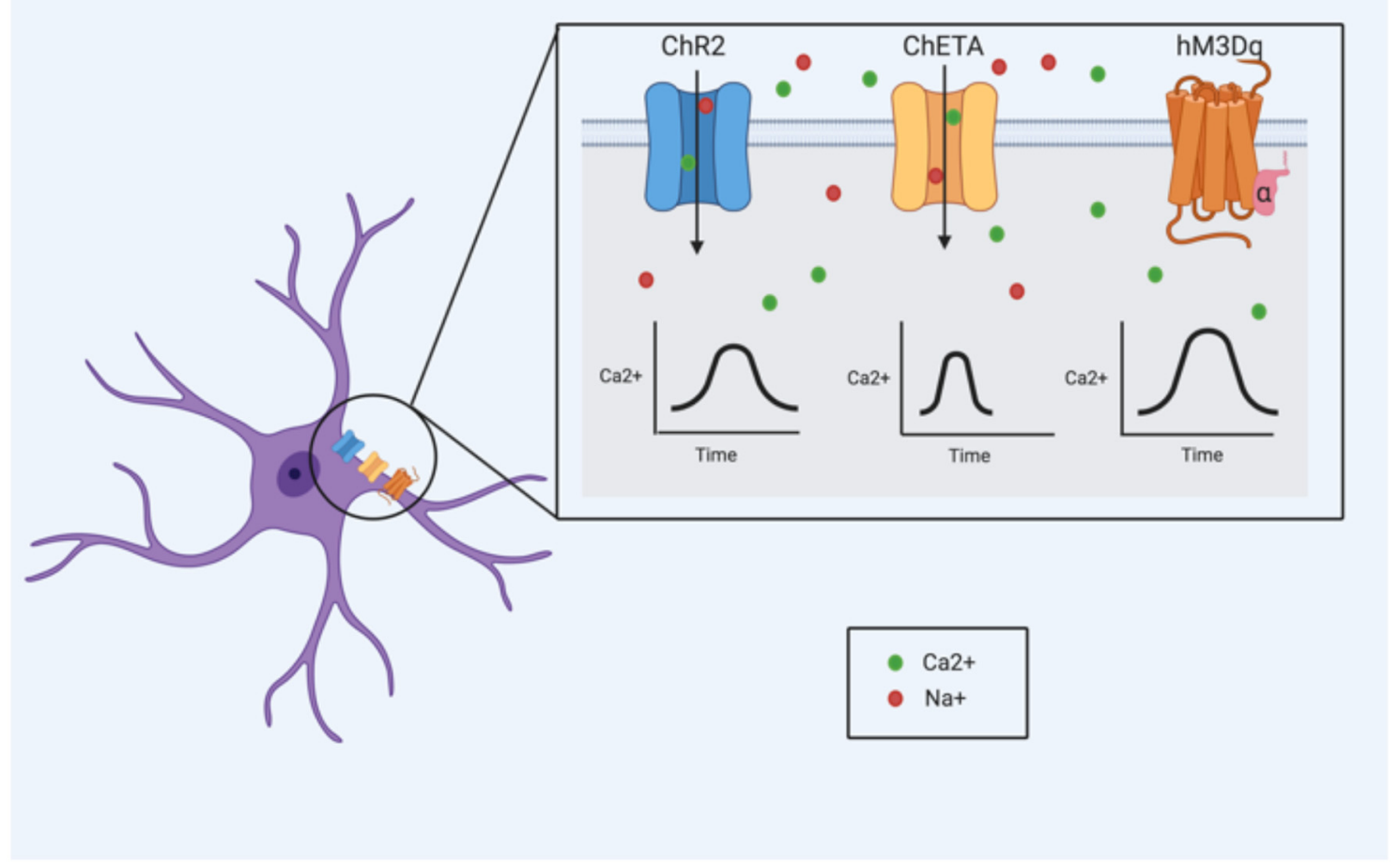

FIGURE 2 | $\mathrm{Ca}^{2+}$ signaling dynamics are influenced by the mechanism of $\mathrm{Ca}^{2+}$ induction. Therefore, opsin and designer receptors exclusively activated by designer drug (DREADD) variants that utilize alternative mechanisms to exert their effects may also show varying $\mathrm{Ca}^{2+}$ responses. These ion channel or receptor kinetics need to be considered when determining the best approach for targeting an astrocyte population.

study compared the effects of stimulating hippocampal astrocytes with ChR2 and melanopsin (Mederos et al., 2019). Although melanopsin was more efficient at inducing $\mathrm{Ca}^{2+}$ signals in both the soma and fine processes (referred to as microdomains in this study) of astrocytes, ChR2 stimulation elicited greater amplitude in miniature EPSCs of CA1 pyramidal neurons (Mederos et al., 2019). This effect on synaptic activity also persisted for longer following the ChR2 stimulation. However, these changes were only seen under certain stimulation parameters and not others (Mederos et al., 2019). Despite being "depolarizing" opsins, both melanopsin and $\mathrm{ChR} 2$ elicit different $\mathrm{Ca}^{2+}$ signals and ultimately, different downstream effects. This makes direct comparisons between studies that utilize different opsins difficult and also highlights how opsins may be physiologically relevant for some contexts, but not others. The effects that different opsins have on $\mathrm{Ca}^{2+}$ dynamics may be important for experimental design. Consideration of the effects that different opsins have on intracellular $\mathrm{K}^{+}$and $\mathrm{Na}^{+}$levels is also critical given their roles in numerous astrocyte functions.

In studies where the activity of select cell populations are directly targeted and modulated, it is essential to validate that the expression of the opsin or DREADD is restricted to that population only. Co-localization of the fluorescent tag with a cell-specific marker can confirm the specificity of this expression. Within astroglial cells, $\mathrm{Ca}^{2+}$ signals differ throughout their subcellular compartments, with potentially variable effects. Therefore, the precise subcellular localization of an opsin or DREADD receptor within an astrocyte may modulate the overall effect on that individual cell. Moving forward, it may be important to validate whether the subcellular expression does indeed alter functional outcomes, and what those functional outcomes might be. The physiological relevance of these technologies may therefore be better understood with the full knowledge of the cellular distribution of opsins and DREADD receptors in astrocytes.

Through gap junction proteins, astrocytes can create a network of interconnected cells. Several studies have demonstrated that calcium waves initiated in one astrocyte can propagate to others surrounding it (Verkhratsky, 2006). This phenomenon was first observed from in vitro culture work; these results were later confirmed in acute slices (Dani et al., 1992; Konietzko and Müller, 1994), and eventually with in vivo experiments (Kuga et al., 2011). Similar effects have been noted with $\mathrm{Na}^{+}$signals; several studies have demonstrated the ability of astrocytes to propagate these signals from one cell to another via these gap junctions (Rose and Ransom, 1997; Bernardinelli et al., 2004; Langer et al., 2012; Augustin et al., 2016; MoshrefiRavasdjani et al., 2017). What this means for studies in which 


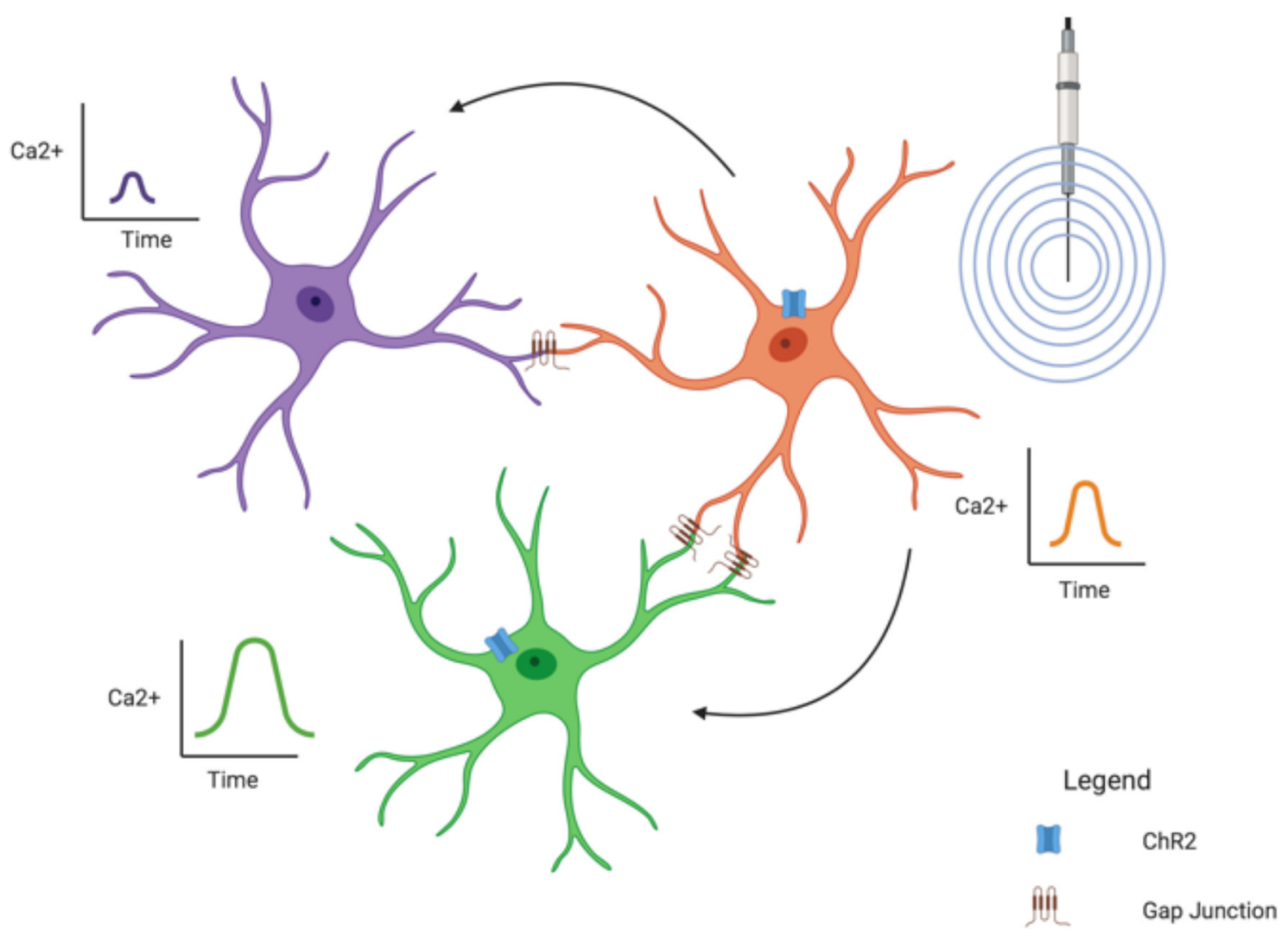

FIGURE 3 | The glial syncytium may impact the physiological effects of optogenetics and DREADD technologies (optogenetics illustrated above). The connection of astrocytes via gap junction proteins might produce a "double stimulation" or "spillover" effect. In the diagram above, only a portion of the astrocytes (orange and green) express the opsin of interest. The green astrocyte is first stimulated following the introduction of light into the system, and then again when a neighboring astrocyte (orange) is also stimulated, generating a larger than anticipated response. In the case of "spillover," astrocytes that do not express the opsin or DREADD in use nonetheless become "stimulated" from the activation of its neighboring astrocytes. In the image above, the purple astrocyte still exhibits a response following light stimulation despite the lack of ChR2 expression. The "stimulation" arises from the influence of the connected astrocyte (orange) that is stimulated via its ChR2 ion channels.

astroglial cells are manipulated remains to be seen. In most cases, it is unlikely that all astrocytes in a region of interest express the opsin or DREADD receptor. However, via the glial syncytium, it is possible that "activation" of one astrocyte leads to the activation of surrounding astrocytes. In one study, $\mathrm{Ca}^{2+}$ changes were restricted to only astrocytes expressing the opsin (Arch; Poskanzer and Yuste, 2016). However, only $\mathrm{Ca}^{2+}$ changes were measured, and it is possible that other ions/substrates were altered in coupled, $\mathrm{Arch}^{-}$astrocytes. The potential of $\mathrm{Ca}^{2+}$ or other compounds to move from a stimulated astrocyte to its coupled neighbors needs to be studied under other parameters and with different opsin and DREADD variants. How far this effect could spread would likely depend on the extent of astroglial coupling within regions of interest. In vivo imaging of astrocytes during optogenetic stimulation or DREADD activation may help answer these questions.

Importantly, whether this stimulation of one astrocyte leads to the stimulation of other astrocytes will likely also depend on the integrity of the glial syncytium. The Murphy-Royal et al.'s (2020) study described in the previous section found acute stress was sufficient to reduce the amount of gap junction coupling between astrocytes. Therefore, the paradigm under which optogenetics is employed could significantly impact the extent to which the optogenetic stimulation of astrocytes "spreads" or influences astrocytes of the neighboring area. As such, optogenetic stimulation of astrocytes under normal, physiological conditions, when presumably the glial syncytium is intact, may have a greater impact than stimulation in a model of stress or injury where this may not be the case.

Another consideration is the potential of "double stimulation." An astroglial cell stimulated via optogenetics or DREADDs could, through gap junction coupling, stimulate a neighboring astrocyte. If this astrocyte is also stimulated via optogenetic or DREADD technology, this may result in a "double stimulation" effect, leading to a response much greater than physiological conditions. A stronger response could mean the stimulated astrocytes influence neighboring cells, in particular, neurons, in a manner incompatible with a normal physiological response. The strength of the glial syncytium in a given area may dictate the likelihood that this happens, further highlighting 
the necessity of understanding the heterogeneous nature of astrocyte physiology and connectivity. As stress can influence the integrity of the glial syncytium (Murphy-Royal et al., 2020), this (potential) issue of "double stimulation" may be less of a concern in paradigms where the astrocytes are stimulated under conditions of stress or injury/disease. This demonstrates the importance of pilot studies to understand how optogenetics and DREADDs impact astoglial activity (see Figure 3).

In optogenetic and DREADD studies involving neurons, their heterogeneous nature is taken into consideration when designing experiments. The opsin variant or DREADD, for example, that is used to stimulate or inhibit a GABAergic neuron may differ from that of a glutamatergic or dopaminergic one. This same consideration needs to be taken for astrocytes. The literature shows that like neurons, astrocytes are a completely heterogenous population. They differ extensively in their morphology, electrophysiology, protein expression, and function. These subtypes are important to consider as each type may not respond in the same manner to different opsin and DREADD variants. Moving forward, understanding these subtypes will be crucial for informing experimental design. Greater knowledge of astrocyte electrophysiology will be an essential component for characterizing these astrocyte subtypes.

Similarly, in neurons, the use of an opsin or DREADD variant largely depends on the desired effect that researchers are looking to elicit. In neurons, these desired effects are typically the facilitation or inhibition of an action potential, driven by depolarization or hyperpolarization of the targeted cell. However, with astrocytes, a depolarization or hyperpolarization will not elicit these same effects. As such, understanding astrocyte physiology, and the heterogeneous nature of this physiology, will be critical for making knowledgeable and appropriate decisions regarding experimental design.

\section{CONCLUSION}

The research over the past few decades has demonstrated that astrocytes are a more "excitable" and heterogenous cell

\section{REFERENCES}

Abney, E. R., Bartlett, P. P., and Raff, M. C. (1981). Astrocytes, ependymal cells, and oligodendrocytes develop on schedule in dissociated cell cultures of embryonic rat brain. Dev. Biol. 83, 301-310. doi: 10.1016/0012-1606(81) 90476-0

Agarwal, A., Wu, P.-H., Hughes, E. G., Fukaya, M., Tischfield, M. A., Langseth, A. J., et al. (2017). Transient opening of the mitochondrial permeability transition pore induces microdomain calcium transients in astrocyte processes. Neuron 93, 587.e7-605.e7. doi: 10.1016/j.neuron.2016. 12.034

Aguado, F., Espinosa-Parrilla, J. F., Carmona, M. A., and Soriano, E. (2002). Neuronal activity regulates correlated network properties of spontaneous calcium transients in astrocytes in situ. J. Neurosci. 22, 9430-9444. doi: 10.1523/JNEUROSCI.22-21-09430.2002

Agulhon, C., Petravicz, J., McMullen, A. B., Sweger, E. J., Minton, S. K., Taves, S. R., et al. (2008). What is the role of astrocyte calcium in neurophysiology? Neuron 59, 932-946. doi: 10.1016/j.neuron.2008.09.004

Alexander, G. M., Rogan, S. C., Abbas, A. I., Armbruster, B. N., Pei, Y., Allen, J. A., et al. (2009). Remote control of neuronal activity in transgenic mice expressing population than previously believed. They differ in morphology, function, and physiology, though the extent of physiological diversity has not been fully characterized. In this review, we discussed how astrocytes exhibit a wide range of basic electrophysiological properties under basal conditions. The mechanisms underlying homeostatic potassium regulation vary between and within brain regions, as do the specific variants of $\mathrm{K}^{+}$channels that mediate this $\mathrm{K}^{+}$buffering. Likewise, the $\mathrm{Na}^{+}$currents and $\mathrm{Na}^{+}$channel subtypes that mediate those currents are also heterogenous in astrocytes. $\mathrm{Ca}^{2+}$ signaling, a key component of astrocyte physiology is also highly variable. There are several different types of $\mathrm{Ca}^{2+}$ waves present in astrocytes, and these vary considerably amongst astrocytes across the CNS. Precisely how all these electrophysiological differences influence surrounding network activity is still unclear, but there is some evidence to indicate that they do. For example, asynchronous $\mathrm{Ca}^{2+}$ microdomains in astrocyte processes likely provides a synapse-specific influence of network activity. This heterogeneity is important to consider for optogenetic and DREADD technologies so that the limitations and generalization of each study can be fully assessed.

Astrocytes represent a very heterogenous cell population; we have highlighted some of the vast differences between the electrophysiological properties of these cells. Despite the available evidence, there is still a substantial amount of research needed in order to truly understand the extensive diversity of this unique cell population.

\section{AUTHOR CONTRIBUTIONS}

JM, MH, CR, and NS wrote the manuscript. JM and NS made the figures. All authors contributed to the article and approved the submitted version.

\section{FUNDING}

This work was supported by a Canadian Institute of Health Research Canada Research Chair to NS.

evolved G protein-coupled receptors. Neuron 63, 27-39. doi: 10.1016/j.neuron. 2009.06.014

Allen, N. J., and Eroglu, C. (2017). Cell biology of astrocyte-synapse interactions. Neuron 96, 697-708. doi: 10.1016/j.neuron.2017.09.056

Anderson, S., Brismar, T., and Hansson, E. (1995). Effect of external $\mathrm{K}^{+}, \mathrm{Ca}^{2+}$ and $\mathrm{Ba}^{2+}$ on membrane potential and ionic conductance in rat astrocytes. Cell Mol. Neurobiol. 15, 439-450. doi: 10.1007/BF02071879

Annunziato, L., Boscia, F., and Pignataro, G. (2013). Ionic transporter activity in astrocytes, microglia, and oligodendrocytes during brain ischemia. J. Cereb. Blood Flow Metab. 33, 969-982. doi: 10.1038/jcbfm.2013.44

Araque, A., Carmignoto, G., Haydon, P. G., Oliet, S. H. R., Robitaille, R., and Volterra, A. (2014). Gliotransmitters travel in space and time. Neuorn 81, 728-739. doi: 10.1016/j.neuron.2014.02.007

Asada, A., Ujita, S., Nakayama, R., Oba, S., Ishii, S., Matsuki, N., et al. (2015). Subtle modulation of ongoing calcium dynamics in astrocytic microdomains by sensory inputs. Physiol. Rep. 3:e12454. doi: 10.14814/phy2. 12454

Augustin, V., Bold, C., Wadle, S. L., Langer, J., Jabs, R., Philippot, C., et al. (2016). Functional anisotropic panglial networks in the lateral superior olive. Glia 64, 1892-1911. doi: 10.1002/glia.23031 
Balachandar, L., Montejo, K. A., Castano, E., Perez, M., Moncion, C., Chambers, J. W., et al. (2020). Simultaneous $\mathrm{Ca}^{2+}$ imaging and optogenetic stimulation of cortical astrocytes in adult murine brain slices. Curr. Protoc. Neurosci. 94:e110. doi: 10.1002/cpns.110

Bang, J., Kim, H. Y., and Lee, H. (2016). Optogenetic and chemogenetic approaches for studying astrocytes and gliotransmitters. Exp. Neurobiol. 25, 205-221. doi: 10.5607/en.2016.25.5.205

Bayraktar, O. A., Bartels, T., Holmqvist, S., Kleshchevnikov, V., Martirosyan, A., Polioudakis, D., et al. (2020). Astrocyte layers in the mammalian cerebral cortex revealed by a single-cell in situ transcriptomic map. Nat. Neurosci. 23, 500-509. doi: 10.1038/s41593-020-0602-1

Bazargani, N., and Attwell, D. (2016). Astrocyte calcium signaling: the third wave. Nat. Neurosci. 19, 182-189. doi: 10.1038/nn.4201

Bedner, P., Dupper, A., Hüttmann, K., Müller, J., Herde, M. K., Dublin, P., et al. (2015). Astrocyte uncoupling as a cause of human temporal lobe epilepsy. Brain 138, 1208-1222. doi: 10.1093/brain/awv067

Bedner, P., Jabs, R., and Steinhäuser, C. (2020). Properties of human astrocytes and NG2 glia. Glia 68, 756-767. doi: 10.1002/glia.23725

Bekar, L. K., Loewen, M. E., Cao, K., Sun, X., Leis, J., Wang, R., et al. (2005). Complex expression and localization of inactivating Kv channels in cultured hippocampal astrocytes. J. Neurophysiol. 93, 1699-1709. doi: 10.1152/jn.008 50.2004

Bekar, L. K., and Walz, W. (1999). Evidence for chloride ions as intracellular messenger substances in astrocytes. J. Neurophysiol. 82, 248-254. doi: 10.1152/jn.1999.82.1.248

Bellot-Saez, A., Kékesi, O., Morley, J. W., and Buskila, Y. (2017). Astrocytic modulation of neuronal excitability through $\mathrm{K}^{+}$spatial buffering. Neurosci. Biobehav. Rev. 77, 87-97. doi: 10.1016/j.neubiorev.2017.03.002

Benesova, J., Rusnakova, V., Honsa, P., Pivonkova, H., Dzamba, D., Kubista, M., et al. (2012). Distinct expression/function of potassium and chloride channels contributes to the diverse volume regulation in cortical astrocytes of GFAP/EGFP mice. PLoS One 7:e29725. doi: 10.1371/journal.pone.00 29725

Bernardinelli, Y., Magistretti, P. J., and Chatton, J.-Y. (2004). Astrocytes generate $\mathrm{Na}^{+}$-mediated metabolic waves. Proc. Natl. Acad. Sci. U S A 101, 14937-14942. doi: 10.1073/pnas.0405315101

Bindocci, E., Savtchouk, I., Liaudet, N., Becker, D., Carriero, G., and Volterra, A. (2017). Three-dimensional $\mathrm{Ca}^{2+}$ imaging advances understanding of astrocyte biology. Science 356:eaai8185. doi: 10.1126/science.aai8185

Black, J. A., and Waxman, S. G. (2013). Noncanonical roles of voltagegated sodium channels. Neuron 80, 280-291. doi: 10.1016/j.neuron.2013. 09.012

Black, J. A., Westenbroek, R., Minturn, J. E., Ransom, B. R., Catterall, W. A., and Waxman, S. G. (1995). Isoform-specific expression of sodium channels in astrocytes in vitro: immunocytochemical observations. Glia 14, 133-144. doi: 10.1002/glia.440140208

Bolton, S., Greenwood, K., Hamilton, N., and Butt, A. M. (2006). Regulation of the astrocyte resting membrane potential by cyclic AMP and protein kinase A. Glia 54, 316-328. doi: 10.1002/glia.20384

Bonaventura, J., Eldridge, M. A. G., Hu, F., Gomez, J. L., Sanchez-Soto, M., Abramyan, A. M., et al. (2019). High-potency ligands for DREADD imaging and activation in rodents and monkeys. Nat. Commun. 10:4627. doi: 10.1038/s41467-019-12236-z

Bordey, A., Lyons, S. A., Hablitz, J. J., and Sontheimer, H. (2001). Electrophysiological characteristics of reactive astrocytes in experimental cortical dysplasia. J. Neurophysiol. 85, 1719-1731. doi: 10.1152/jn.2001.85.4. 1719

Bordey, A., and Sontheimer, H. (1998). Electrophysiological properties of human astrocytic tumor cells in situ: enigma of spiking glial cells. J. Neurophysiol. 79, 2782-2793. doi: 10.1152/jn.1998.79.5.2782

Bordey, A., and Sontheimer, H. (2000). Ion channel expression by astrocytes in situ: comparison of different CNS regions. Glia 30, 27-38. doi: 10.1002/(sici)1098-1136(200003)30:1<27::aid-glia4>3.0.co;2-\#

Boyden, E. S., Zhang, F., Bamberg, E., Nagel, G., and Deisseroth, K. (2005). Millisecond-timescale, genetically targeted optical control of neural activity. Nat. Neurosci. 8, 1263-1268. doi: 10.1038/nn1525

Brasko, C., Hawkins, V., De La Rocha, I. C., and Butt, A. M. (2017). Expression of Kir4.1 and Kir5.1 inwardly rectifying potassium channels in oligodendrocytes, the myelinating cells of the CNS. Brain Struct. Funct. 222, 41-59. doi: 10.1007/s00429-016-1199-8

Brockhaus, J., and Deitmer, J. W. (2002). Long-lasting modulation of synaptic input to Purkinje neurons by Bergmann glia stimulation in rat brain slices. J. Physiol. 545, 581-593. doi: 10.1113/jphysiol.2002. 028423

Bushong, E. A., Martone, M. E., and Ellisman, M. H. (2004). Maturation of astrocyte morphology and the establishment of astrocyte domains during postnatal hippocampal development. Int. J. Dev. Neurosci. 22, 73-86. doi: 10.1016/j.ijdevneu.2003.12.008

Butt, A. M., and Jennings, J. (1994). Response of astrocytes to $\gamma$-aminobutyric acid in the neonatal rat optic nerve. Neurosci. Lett. 168, 53-56. doi: 10.1016/03043940(94)90414-6

Cahoy, J. D., Emery, B., Kaushal, A., Foo, L. C., Zamanian, J. L., Christopherson, K. S., et al. (2008). A transcriptome database for astrocytes, neurons, and oligodendrocytes: a new resource for understanding brain development and function. J. Neurosci. 28, 264-278. doi: 10.1523/JNEUROSCI. 4178-07.2008

Chai, H., Diaz-castro, B., Shigetomi, E., Monte, E., Octeau, J. C., Yu, X., et al. (2017). Neural circuit-specialized astrocytes: transcriptomic, proteomic, morphological, and functional evidence. Neuron 95, 531-549. doi: 10.1016/j. neuron.2017.06.029

Chen, J., Tan, Z., Zeng, L. I., Zhang, X., He, Y., Gao, W., et al. (2013). Heterosynaptic long-term depression mediated by ATP released from astrocytes. Glia 61, 178-191. doi: 10.1002/glia.22425

Cotrina, M. L., Lin, J. H., López-García, J. C., Naus, C. C., and Nedergaard, M. (2000). ATP-mediated glia signaling. J. Neurosci. 20, 2835-2844. doi: 10.1523/JNEUROSCI.20-08-02835.2000

Dallérac, G., Chever, O., and Rouach, N. (2013). How do astrocytes shape synaptic transmission? Insights from electrophysiology. Front. Cell. Neurosci. 7:159. doi: 10.3389/fncel.2013.00159

Dani, J. W., Chernjavsky, A., and Smith, S. J. (1992). Neuronal activity triggers calcium waves in hippocampal astrocyte networks. Neuron 8, 429-440. doi: 10.1016/0896-6273(92)90271-e

de Lanerolle, N., and Lee, T.-S. (2005). New facets of the neuropathology and molecular profile of human temporal lobe epilepsy. Epilepsy Behav. 7, 190-203. doi: 10.1016/j.yebeh.2005.06.003

Deemyad, T., Lüthi, J., and Spruston, N. (2018). Astrocytes integrate and drive action potential firing in inhibitory subnetworks. Nat. Commun. 9:4336. doi: 10.1038/s41467-018-06338-3

Dossi, E., Vasile, F., and Rouach, N. (2018). Human astrocytes in the diseased brain. Brain Res. Bull. 136, 139-156. doi: 10.1016/j.brainresbull.2017. 02.001

Du, Y., Kiyoshi, C. M., Wang, Q., Wang, W., Ma, B., Alford, C. C., et al. (2016). Genetic deletion of TREK-1 or TWIK-1/TREK-1 potassium channels does not alter the basic electrophysiological properties of mature hippocampal astrocytes in situ. Front. Cell. Neurosci. 10:13. doi: 10.3389/fncel.2016.00013

Du, Y., Ma, B., Kiyoshi, C. M., Alford, C. C., Wang, W., and Zhou, M. (2015). Freshly dissociated mature hippocampal astrocytes exhibit passive membrane conductance and low membrane resistance similarly to syncytial coupled astrocytes. J. Neurophysiol. 113, 3744-3750. doi: 10.1152/jn.002 06.2015

El-Gaby, M., Zhang, Y., Wolf, K., Schwiening, C. J., Paulsen, O., and Shipton, O. A. (2016). Archaerhodopsin selectively and reversibly silences synaptic transmission through altered pH. Cell Rep. 16:2259. doi: 10.1016/j. celrep.2016.07.057

Fernandez, F. R., Noueihed, J., and White, J. A. (2019). Voltage-dependent membrane properties shape the size but not the frequency content of spontaneous voltage fluctuations in layer $2 / 3$ somatosensory cortex. J. Neurosci. 39, 2221-2237. doi: 10.1523/JNEUROSCI.1648-18.2019

Figueiredo, M., Lane, S., Stout, R. F., Liu, B., Parpura, V., Teschemacher, A. G., et al. (2014). Comparative analysis of optogenetic actuators in cultured astrocytes. Cell Calcium 56, 208-214. doi: 10.1016/j.ceca.2014.07.007

Freeman, M. R. (2010). Specification and morphogenesis of astrocytes. Science 330, 774-778. doi: 10.1126/science.1190928

Gnatenco, C., Han, J., Snyder, A. K., and Kim, D. (2002). Functional expression of TREK-2 $\mathrm{K}^{+}$channel in cultured rat brain astrocytes. Brain Res. 931, 56-67. doi: 10.1016/s0006-8993(02)02261-8 
Gourine, A. V., Kasymov, V., Marina, N., Tang, F., Figueiredo, M. F., Lane, S., et al. (2010). Astrocytes control breathing through $\mathrm{pH}$-dependent release of ATP. Science 329, 571-575. doi: 10.1126/science.1190721

Guatteo, E., Stanness, K. A., and Janigro, D. (1996). Hyperpolarization-activated ion currents in cultured rat cortical and spinal cord astrocytes. Glia 16, 196-209. doi: 10.1002/(SICI)1098-1136(199603)16:3<196::AID-GLIA2>3. $0 . \mathrm{CO} ; 2-0$

Gunaydin, L. A., Yizhar, O., Berndt, A., Sohal, V. S., Deisseroth, K., and Hegemann, P. (2010). Ultrafast optogenetic control. Nat. Neurosci. 13, 387-392. doi: 10.1038/nn.2495

Hashioka, S., Wang, Y. F., Little, J. P., Choi, H. B., Klegeris, A., McGeer, P. L., et al. (2014). Purinergic responses of calcium-dependent signaling pathways in cultured adult human astrocytes. BMC Neurosci. 15:18. doi: 10.1186/14712202-15-18

Haustein, M. D., Kracun, S., Lu, X.-H., Shih, T., Jackson-Weaver, O., Tong, X., et al. (2014). Conditions and constraints for astrocyte calcium signaling in the hippocampal mossy fiber pathway. Neuron 82, 413-429. doi: 10.1016/j.neuron. 2014.02.041

Haydon, P. G., and Carmignoto, G. (2006). Astrocyte control of synaptic transmission and neurovascular coupling. Physiol. Rev. 86, 1009-1031. doi: 10.1152/physrev.00049.2005

Henneberger, C., Papouin, T., Oliet, S. H. R., and Rusakov, D. A. (2010). Longterm potentiation depends on release of D-serine from astrocytes. Nature 463, 232-236. doi: 10.1038/nature08673

Hertz, L., and Chen, Y. (2016). Importance of astrocytes for potassium ion $\left(\mathrm{K}^{+}\right)$ homeostasis in brain and glial effects of $\mathrm{K}^{+}$and its transporters on learning. Neurosci. Biobehav. Rev. 71, 484-505. doi: 10.1016/j.neubiorev.2016.09.018

Hibino, H., Fujita, A., Iwai, K., Yamada, M., and Kurachi, Y. (2004). Differential assembly of inwardly rectifying $\mathrm{K}^{+}$channel subunits, Kir4.1 and Kir5.1, in brain astrocytes. J. Biol. Chem. 279, 44065-44073. doi: 10.1074/jbc. M405985200

Higashi, K., Fujita, A., Inanobe, A., Tanemoto, M., Doi, K., Kubo, T., et al. (2001). An inwardly rectifying $\mathrm{K}^{+}$channel, Kir4.1, expressed in astrocytes surrounds synapses and blood vessels in brain. Am. J. Physiol. Cell Physiol. 281, C922-C931. doi: 10.1152/ajpcell.2001.281.3.C922

Hinterkeuser, S., Schröder, W., Hager, G., Seifert, G., Blümcke, I., Elger, C. E., et al. (2000). Astrocytes in the hippocampus of patients with temporal lobe epilepsy display changes in potassium conductances. Eur. J. Neurosci. 12, 2087-2096. doi: 10.1046/j.1460-9568.2000.00104.x

Holtzclaw, L. A., Pandhit, S., Bare, D. J., Mignery, G. A., and Russell, J. T. (2002). Astrocytes in adult rat brain express type 2 inositol 1,4,5-trisphosphate receptors. Glia 39, 69-84. doi: 10.1002/glia.10085

Isokawa, M., and McKhann, G. M. (2005). Electrophysiological and morphological characterization of dentate astrocytes in the hippocampus. J. Neurobiol. 65, 125-134. doi: 10.1002/neu.20186

Jiang, R., Diaz-Castro, B., Looger, L. L., and Khakh, B. S. (2016). Dysfunctional calcium and glutamate signaling in striatal astrocytes from Huntington's disease model mice. J. Neurosci. 36, 3453-3470. doi: 10.1523/JNEUROSCI. 3693-15.2016

Jiang, R., Haustein, M. D., Sofroniew, M. V., and Khakh, B. S. (2014). Imaging intracellular $\mathrm{Ca}^{2+}$ signals in striatal astrocytes from adult mice using genetically-encoded calcium indicators. J. Vis. Exp. 93:e51972. doi: $10.3791 / 51972$

Kalsi, A. S., Greenwood, K., Wilkin, G., and Butt, A. M. (2004). Kir4.1 expression by astrocytes and oligodendrocytes in CNS white matter: a developmental study in the rat optic nerve. J. Anat. 204, 475-485. doi: 10.1111/j.0021-8782.2004. 00288.x

Kanemaru, K., Sekiya, H., Xu, M., Satoh, K., Kitajima, N., Yoshida, K., et al. (2014). in vivo visualization of subtle, transient, and local activity of astrocytes using an ultrasensitive $\mathrm{Ca}^{2+}$ indicator. Cell Rep. 8, 311-318. doi: 10.1016/j.celrep.2014. 05.056

Kang, J., Jiang, L., Goldman, S. A., and Nedergaard, M. (1998). Astrocyte-mediated potentiation of inhibitory synaptic transmission. Nat. Neurosci. 1, 683-692. doi: $10.1038 / 3684$

Kofuji, P., Biedermann, B., Siddharthan, V., Raap, M., Iandiev, I., Milenkovic, I., et al. (2002). Kir potassium channel subunit expression in retinal glial cells: implications for spatial potassium buffering. Glia 39, 292-303. doi: 10.1002/glia.10112
Khakh, B. S., and Deneen, B. (2019). The emerging nature of astrocyte diversity. Annu. Rev. Neurosci. 42, 187-207. doi: 10.1146/annurev-neuro-070918050443

Kofuji, P., and Newman, E. A. (2004). Potassium buffering in the central nervous system. Neuroscience 129, 1045-1056. doi: 10.1016/j.neuroscience.2004.06.008

Konietzko, U., and Müller, C. M. (1994). Astrocytic dye coupling in rat hippocampus: topography, developmental onset, and modulation by protein kinase C. Hippocampus 4, 297-306. doi: 10.1002/hipo.450040313

Kressin, K., Kuprijanova, E., Jabs, R., Seifert, G., and Steinhäuser, C. (1995). Developmental regulation of $\mathrm{Na}^{+}$and $\mathrm{K}^{+}$conductances in glial cells of mouse hippocampal brain slices. Glia 15, 173-187. doi: 10.1002/glia.440150210

Kuga, N., Sasaki, T., Takahara, Y., Matsuki, N., and Ikegaya, Y. (2011). Large-scale calcium waves traveling through astrocytic networks in vivo. J. Neurosci. 31, 2607-2614. doi: 10.1523/JNEUROSCI.5319-10.2011

Langer, J., and Rose, C. R. (2009). Synaptically induced sodium signals in hippocampal astrocytes in situ. J. Physiol. 587, 5859-5877. doi: 10.1113/jphysiol.2009.182279

Langer, J., Stephan, J., Theis, M., and Rose, C. R. (2012). Gap junctions mediate intercellular spread of sodium between hippocampal astrocytes in situ. Glia 60, 239-252. doi: 10.1002/glia.21259

Lanjakornsiripan, D., Pior, B. J., Kawaguchi, D., Furutachi, S., Tahara, T., Katsuyama, Y., et al. (2018). Layer-specific morphological and molecular differences in neocortical astrocytes and their dependence on neuronal layers. Nat. Commun. 9:1623. doi: 10.1038/s41467-018-03940-3

Li, D., Hérault, K., Isacoff, E. Y., Oheim, M., and Ropert, N. (2012). Optogenetic activation of LiGluR-expressing astrocytes evokes anion channel-mediated glutamate release. J. Physiol. 590, 855-873. doi: 10.1113/jphysiol.2011. 219345

Li, Y.-K., Wang, F., Wang, W., Luo, Y., Wu, P.-F., Xiao, J.-L., et al. (2012). Aquaporin-4 deficiency impairs synaptic plasticity and associative fear memory in the lateral amygdala: involvement of downregulation of glutamate transporter-1 expression. Neuropsychopharmacology 37, 1867-1878. doi: 10.1038/npp.2012.34

Liddelow, S. A., Guttenplan, K. A., Clarke, L. E., Bennett, F. C., Bohlen, C. J., Schirmer, L., et al. (2017). Neurotoxic reactive astrocytes are induced by activated microglia. Nature 541, 481-487. doi: 10.1038/nature21029

Liedtke, W., Edelmann, W., Bieri, P. L., Chiu, F. C., Cowan, N. J., Kucherlapati, R., et al. (1996). GFAP is necessary for the integrity of CNS white matter architecture and long-term maintenance of myelination. Neuron 17, 607-615. doi: 10.1016/s0896-6273(00)80194-4

Ma, B., Xu, G., Wang, W., Enyeart, J. J., and Zhou, M. (2014). Dual patch voltage clamp study of low membrane resistance astrocytes in situ. Mol. Brain 7:18. doi: 10.1186/1756-6606-7-18

Mahmoud, S., Gharagozloo, M., Simard, C., and Gris, D. (2019). Astrocytes maintain glutamate homeostasis in the CNS by controlling the balance between glutamate uptake and release. Cells 8:184. doi: 10.3390/cells8 020184

Manvich, D. F., Webster, K. A., Foster, S. L., Farrell, M. S., Ritchie, J. C., Porter, J. H., et al. (2018). The DREADD agonist clozapine N-oxide (CNO) is reverse-metabolized to clozapine and produces clozapine-like interoceptive stimulus effects in rats and mice. Sci. Rep. 8:3840. doi: 10.1038/s41598-01822116-Z

Mederos, S., Hernández-Vivanco, A., Ramírez-Franco, J., Martín-Fernández, M., Navarrete, M., Yang, A., et al. (2019). Melanopsin for precise optogenetic activation of astrocyte-neuron networks. Glia 67, 915-934. doi: 10.1002/glia. 23580

Mi Hwang, E., Kim, E., Yarishkin, O., Ho Woo, D., Han, K.-S., Park, N., et al. (2014). A disulphide-linked heterodimer of TWIK-1 and TREK-1 mediates passive conductance in astrocytes. Nat. Commun. 5:3227. doi: $10.1038 /$ ncomms 4227

Miller, S. J. (2018). Astrocyte heterogeneity in the adult central nervous system. Front. Cell. Neurosci. 12:401. doi: 10.3389/fncel.2018.00401

Mishima, T., and Hirase, H. (2010). in vivo intracellular recording suggests that gray matter astrocytes in mature cerebral cortex and hippocampus are electrophysiologically homogeneous. J. Neurosci. 30, 3093-3100. doi: 10.1523/JNEUROSCI.5065-09.2010

Moore, Y. E., Conway, L. C., Wobst, H. J., Brandon, N. J., Deeb, T. Z., and Moss, S. J. (2019). Developmental regulation of KCC2 phosphorylation 
has long-term impacts on cognitive function. Front. Mol. Neurosci. 12:173. doi: 10.3389/fnmol.2019.00173

Moshrefi-Ravasdjani, B., Hammel, E. L., Kafitz, K. W., and Rose, C. R. (2017). Astrocyte sodium signalling and panglial spread of sodium signals in brain white matter. Neurochem. Res. 42, 2505-2518. doi: 10.1007/s11064-017 $-2197-9$

Murphy-Royal, C., Johnston, A. D., Boyce, A. K. J., Diaz-Castro, B., Institoris, A., Peringod, G., et al. (2020). Stress gates an astrocytic energy reservoir to impair synaptic plasticity. Nat. Commun. 11:2014. doi: 10.1038/s41467-02015778-9

Nagel, G., Szellas, T., Huhn, W., Kateriya, S., Adeishvili, N., Berthold, P., et al. (2003). Channelrhodopsin-2, a directly light-gated cation-selective membrane channel. Proc. Natl. Acad. Sci. U S A 100, 13940-13945. doi: 10.1073/pnas. 1936192100

Navarrete, M., Perea, G., Fernandez de Sevilla, D., Gómez-Gonzalo, M., Núñez, A., Martín, E. D., et al. (2012). Astrocytes mediate in vivo cholinergic-induced synaptic plasticity. PLoS Biol. 10:e1001259. doi: 10.1371/journal.pbio.10 01259

Navarrete, M., Perea, G., Maglio, L., Pastor, J., Garcia de Sola, R., and Araque, A. (2013). Astrocyte calcium signal and gliotransmission in human brain tissue. Cereb. Cortex 23, 1240-1246. doi: 10.1093/cercor/bhs122

Neprasova, H., Anderova, M., Petrik, D., Vargova, L., Kubinova, S., Chvatal, A., et al. (2007). High extracellular $\mathrm{K}^{+}$evokes changes in voltage-dependent $\mathrm{K}^{+}$ and $\mathrm{Na}^{+}$currents and volume regulation in astrocytes. Pflugers Arch. 453, 839-849. doi: 10.1007/s00424-006-0151-9

Nett, W. J., Oloff, S. H., and McCarthy, K. D. (2002). Hippocampal astrocytes in situ exhibit calcium oscillations that occur independent of neuronal activity. J. Neurophysiol. 87, 528-537. doi: 10.1152/jn.002 68.2001

Newman, L. A., Walker, M. T., Brown, R. L., Cronin, T. W., and Robinson, P. R. (2003). Melanopsin forms a functional short-wavelength photopigment. Biochemistry 42, 12734-12738. doi: 10.1021/bi035418z

O'Connor, E. R., Sontheimer, H., Spencer, D. D., and De Lanerolle, N. C. (1998). Astrocytes from exhibit human hippocampal epileptogenic foci action potential-like responses. Epilepsia 39, 347-354. doi: 10.1093/cid/ ciab196

Oberheim, N. A., Takano, T., Han, X., He, W., Lin, J. H. C., Wang, F., et al. (2009). Uniquely hominid features of adult human astrocytes. J. Neurosci. 29, 3276-3287. doi: 10.1523/JNEUROSCI.4707-08.2009

Oberheim, N. A., Wang, X., Goldman, S., and Nedergaard, M. (2006). Astrocytic complexity distinguishes the human brain. Trends Neurosci. 29, 547-553. doi: 10.1016/j.tins.2006.08.004

Olsen, M. L., Higashimori, H., Campbell, S. L., Hablitz, J. J., and Sontheimer, H. (2006). Functional expression of Kir4.1 channels in spinal cord astrocytes. Glia 53, 516-528. doi: 10.1002/glia.20312

Orkand, R. K., Nicholls, J. G., and Kuffler, S. W. (1966). Effect of nerve impulses on the membrane potential of glial cells in the central nervous system of amphibia. J. Neurophysiol. 29, 788-806. doi: 10.1152/jn.1966.29. 4.788

Otsu, Y., Couchman, K., Lyons, D. G., Collot, M., Agarwal, A., Mallet, J.-M., et al. (2015). Calcium dynamics in astrocyte processes during neurovascular coupling. Nat. Neurosci. 18, 210-218. doi: 10.1038/nn.3906

Paco, S., Hummel, M., Plá, V., Sumoy, L., and Aguado, F. (2016). Cyclic AMP signaling restricts activation and promotes maturation and antioxidant defenses in astrocytes. BMC Genomics 17:304. doi: 10.1186/s12864-0162623-4

Pappalardo, L. W., Black, J. A., and Waxman, S. G. (2016). Sodium channels in astroglia and microglia. Glia 64, 1628-1645. doi: 10.1002/glia. 22967

Pappalardo, L. W., Samad, O. A., Black, J. A., and Waxman, S. G. (2014). Voltage-gated sodium channel Nav 1.5 contributes to astrogliosis in an in vitro model of glial injury via reverse $\mathrm{Na}^{+} / \mathrm{Ca}^{2+}$ exchange. Glia $62,1162-1175$. doi: 10.1002/glia.22671

Pappalardo, L. W., Samad, O. A., Liu, S., Zwinger, P. J., Black, J. A., and Waxman, S. G. (2018). Nav1.5 in astrocytes plays a sex-specific role in clinical outcomes in a mouse model of multiple sclerosis. Glia 66, 2174-2187. doi: 10.1002/glia.23470
Parpura, V., Basarsky, T. A., Liu, F., Jeftinija, K., Jeftinija, S., and Haydon, P. G. (1994). Glutamate-mediated astrocyte-neuron signalling. Nature 369, 744-747. doi: $10.1038 / 369744 \mathrm{a} 0$

Parpura, V., Grubišić, V., and Verkhratsky, A. (2011). Ca ${ }^{2+}$ sources for the exocytotic release of glutamate from astrocytes. Biochim. Biophys. Acta 1813, 984-991. doi: 10.1016/j.bbamcr.2010.11.006

Parpura, V., and Verkhratsky, A. (2012). Homeostatic function of astrocytes: $\mathrm{Ca}^{2+}$ and $\mathrm{Na}^{+}$signalling. Transl. Neurosci. 3, 334-344. doi: 10.2478/s13380-0120040-y

Parri, H. R., Gould, T. M., and Crunelli, V. (2001). Spontaneous astrocytic Ca ${ }^{2+}$ oscillations in situ drive NMDAR-mediated neuronal excitation. Nat. Neurosci. 4, 803-812. doi: 10.1038/90507

Pasti, L., Volterra, A., Pozzan, T., and Carmignoto, G. (1997). Intracellular calcium oscillations in astrocytes: a highly plastic, bidirectional form of communication between neurons and astrocytes in situ. J. Neurosci. 17, 7817-7830. doi: 10.1523/JNEUROSCI.17-20-07817.1997

Perea, G., Yang, A., Boyden, E. S., and Sur, M. (2014). Optogenetic astrocyte activation modulates response selectivity of visual cortex neurons in vivo. Nat. Commun. 5:3262. doi: 10.1038/ncomms4262

Perez, E. J., Tapanes, S. A., Loris, Z. B., Balu, D. T., Sick, T. J., Coyle, J. T., et al. (2017). Enhanced astrocytic d-serine underlies synaptic damage after traumatic brain injury. J. Clin. Invest. 127, 3114-3125. doi: 10.1172/JCI 92300

Petravicz, J., Fiacco, T. A., and McCarthy, K. D. (2008). Loss of IP3 receptordependent $\mathrm{Ca}^{2+}$ increases in hippocampal astrocytes does not affect baseline CA1 pyramidal neuron synaptic activity. J. Neurosci. 28, 4967-4973. doi: 10.1523/JNEUROSCI.5572-07.2008

Poopalasundaram, S., Knott, C., Shamotienko, O. G., Foran, P. G., Dolly, J. O., Ghiani, C. A., et al. (2000). Glial heterogeneity in expression of the inwardly rectifying $\mathrm{K}^{+}$channel, Kir4.1, in adult rat CNS. Glia 30, 362-372. doi: 10.1002/(sici)1098-1136(200006)30:4<362::aid-glia50>3.0.co;2-4

Porter, J. T., and McCarthy, K. D. (1995). GFAP-positive hippocampal astrocytes in situ respond to glutamatergic neuroligands with increases in $\left[\mathrm{Ca}^{2+}\right] \mathrm{i}$. Glia 13, 101-112. doi: 10.1002/glia.440130204

Poskanzer, K. E., and Yuste, R. (2016). Astrocytes regulate cortical state switching in vivo. Proc. Natl. Acad. Sci. U S A 113, E2675-E2684. doi: 10.1073/pnas. 1520759113

Prebil, M., Jensen, J., Zorec, R., and Kreft, M. (2011). Astrocytes and energy metabolism. Arch. Physiol. Biochem. 117, 64-69. doi: 10.3109/13813455.2010. 539616

Preussat, K., Beetz, C., Schrey, M., Kraft, R., Wölfl, S., Kalff, R., et al. (2003). Expression of voltage-gated potassium channels Kv1.3 and Kv1.5 in human gliomas. Neurosci. Lett. 346, 33-36. doi: 10.1016/s0304-3940(03) 00562-7

Qian, X., Shen, Q., Goderie, S. K., He, W., Capela, A., Davis, A. A., et al. (2000). Timing of CNS cell generation: a programmed sequence of neuron and glial cell production from isolated murine cortical stem cells. Neuron 28, 69-80. doi: 10.1016/s0896-6273(00)00086-6

Qiao, X., Werkman, T. R., Gorter, J. A., Wadman, W. J., and van Vliet, E. A. (2013). Expression of sodium channel $\alpha$ subunits 1.1, 1.2 and 1.6 in rat hippocampus after kainic acid-induced epilepsy. Epilepsy Res. 106, 17-28. doi: 10.1016/j. eplepsyres.2013.06.006

Reese, K. A., and Caldwell, J. H. (1999). Immunocytochemical localization of $\mathrm{NaCh} 6$ in cultured spinal cord astrocytes. Glia 26, 92-96. doi: 10.1002/(sici)1098-1136(199903)26:1<92::aid-glia10>3.0.co;2-4

Rose, C. R., and Ransom, B. R. (1997). Gap junctions equalize intracellular $\mathrm{Na}^{+}$concentration in astrocytes. Glia 20, 299-307. doi: 10.1002/(sici)10981136(199708)20:4<299::aid-glia3>3.0.co;2-1

Roth, B. L. (2016). DREADDs for neuroscientists. Neuron 89, 683-694 doi: 10.1016/j.neuron.2016.01.040

Rouach, N., Koulakoff, A., Abudara, V., Willecke, K., and Giaume, C. (2008). Astroglial metabolic networks sustain hippocampal synaptic transmission. Science 322, 1551-1555. doi: 10.1126/science.1164022

Rungta, R. L., Bernier, L.-P., Dissing-Olesen, L., Groten, C. J., LeDue, J. M., Ko, R., et al. (2016). $\mathrm{Ca}^{2+}$ transients in astrocyte fine processes occur via $\mathrm{Ca}^{2+}$ influx in the adult mouse hippocampus. Glia 64, 2093-2103. doi: 10.1002/glia. 23042 
Rurak, G. M., Simard, S., Charih, F., Van Geel, A., Stead, J., Woodside, B., et al. (2020). Translatomic database of cortical astroglia across male and female mouse development reveals two distinct developmental phenotypes. bioRxiv [Preprint]. doi: 10.1101/681684

Ryoo, K., and Park, J.-Y. (2016). Two-pore domain potassium channels in astrocytes. Exp. Neurobiol. 25, 222-232. doi: 10.5607/en.2016. 25.5.222

Schaller, K. L., and Caldwell, J. H. (2003). Expression and distribution of voltage-gated sodium channels in the cerebellum. Cerebellum 2, 2-9. doi: 10.1080/14734220309424

Schröder, W., Hager, G., Kouprijanova, E., Weber, M., Schmitt, A. B., Seifert, G., et al. (1999). Lesion-induced changes of electrophysiological properties in astrocytes of the rat dentate gyrus. Glia 28, 166-174. doi: 10.1002/(sici)10981136(199911)28:2<166::aid-glia8 > 3.0.co;2-t

Seifert, G., Hüttmann, K., Binder, D. K., Hartmann, C., Wyczynski, A., Neusch, C., et al. (2009). Analysis of astroglial $\mathrm{K}^{+}$channel expression in the developing hippocampus reveals a predominant role of the Kir4.1 subunit. J. Neurosci. 29, 7474-7488. doi: 10.1523/JNEUROSCI.3790-08.2009

Shen, Y., Kyu Han, S., and Dong Ryu, P. (2018). Comparison of electrophysiological properties of two types of pre-sympathetic neurons intermingled in the hypothalamic paraventricular nucleus. J. Vet. Sci. 19, 483-491. doi: 10.4142/jvs.2018.19.4.483

Sheppard, C. A., Simpson, P. B., Sharp, A. H., Nucifora, F. C., Ross, C. A., Lange, G. D., et al. (2002). Comparison of type 2 inositol 1,4,5-trisphosphate receptor distribution and subcellular $\mathrm{Ca}^{2+}$ release sites that support $\mathrm{Ca}^{2+}$ waves in cultured astrocytes. J. Neurochem. 68, 2317-2327. doi: 10.1046/j.14714159.1997.68062317.x

Sherwood, M. W., Arizono, M., Hisatsune, C., Bannai, H., Ebisui, E., Sherwood, J. L., et al. (2017). Astrocytic IP 3 Rs: contribution to $\mathrm{Ca}^{2+}$ signalling and hippocampal LTP. Glia 65, 502-513. doi: 10.1002/glia. 23107

Shigetomi, E., Bushong, E. A., Haustein, M. D., Tong, X., Jackson-Weaver, O., Kracun, S., et al. (2013). Imaging calcium microdomains within entire astrocyte territories and endfeet with GCaMPs expressed using adeno-associated viruses. J. Gen. Physiol. 141, 633-647. doi: 10.1085/jgp.201210949

Shigetomi, E., Saito, K., Sano, F., and Koizumi, S. (2019). Aberrant calcium signals in reactive astrocytes: a key process in neurological disorders. Int. J. Mol. Sci. 20:996. doi: 10.3390/ijms20040996

Sloan, S. A., and Barres, B. A. (2014). Mechanisms of astrocyte development and their contributions to neurodevelopmental disorders. Curr. Opin. Neurobiol. 27, 75-81. doi: 10.1016/j.conb.2014.03.005

Smart, S. L., Bosma, M. M., and Tempel, B. L. (1997). Identification of the delayed rectifier potassium channel, Kv1.6, in cultured astrocytes. Glia 20, 127-134. doi: 10.1002/(sici)1098-1136(199706)20:2<127::aid-glia4>3.0.co;2-6

Smith, K. S., Bucci, D. J., Luikart, B. W., and Mahler, S. V. (2016). DREADDS: use and application in behavioral neuroscience. Behav. Neurosci. 130, 137-155. doi: 10.1037/bne0000135

Smith, N. A., Kress, B. T., Lu, Y., Chandler-Militello, D., Benraiss, A., and Nedergaard, M. (2018). Fluorescent $\mathrm{Ca}^{2+}$ indicators directly inhibit the Na,K-ATPase and disrupt cellular functions. Sci. Signal. 11:eaal2039. doi: 10.1126/scisignal.aal2039

Sofroniew, M. V., and Vinters, H. V. (2010). Astrocytes: biology and pathology. Acta Neuropathol. 119, 7-35. doi: 10.1007/s00401-0090619-8

Somjen, G. G. (1979). Extracellular potassium in the mammalian central nervous system. Annu. Rev. Physiol. 41, 159-177. doi: 10.1146/annurev.ph.41.030179. 001111

Sontheimer, H. (1994). Voltage-dependent ion channels in glial cells. Glia 11, 156-172. doi: 10.1002/glia.440110210

Sontheimer, H., Fernandez-Marques, E., Ullrich, I. N., Pappas, C. A., and Waxman, S. G. (1994). Astrocyte $\mathrm{Na}^{+}$channels are required for maintenance of $\mathrm{Na}^{+} / \mathrm{K}^{+}$-ATPase activity. J. Neurosci. 14, 2464-2475. doi: 10.1523/JNEUROSCI.14-05-02464.1994

Sontheimer, H., Minturn, J. E., Black, J. A., Ransom, B. R., and Waxman, S. G. (1991). Two types of $\mathrm{Na}^{+}$-currents in cultured rat optic nerve astrocytes: changes with time in culture and with age of culture derivation. J. Neurosci. Res. 30, 275-287. doi: 10.1002/jnr.490300202
Sontheimer, H., and Waxman, S. G. (1992). Ion channels in spinal cord astrocytes in vitro. II. Biophysical and pharmacological analysis of two $\mathrm{Na}^{+}$current types. J. Neurophysiol. 68, 1001-1011. doi: 10.1152/jn.1992.68.4.1001

Srinivasan, R., Huang, B. S., Venugopal, S., Johnston, A. D., Chai, H., Zeng, H., et al. (2015). $\mathrm{Ca}^{2+}$ signaling in astrocytes from Ip3r2-/- mice in brain slices and during startle responses in vivo. Nat. Neurosci. 18, 708-717. doi: $10.1038 / \mathrm{nn} .4001$

Stephan, J., Haack, N., Kafitz, K. W., Durry, S., Koch, D., Hochstrate, P., et al. (2012). Kir4.1 channels mediate a depolarization of hippocampal astrocytes under hyperammonemic conditions in situ. Glia 60, 965-978. doi: 10.1002/glia. 22328

Stobart, J. L., Ferrari, K. D., Barrett, M. J. P., Glück, C., Stobart, M. J., Zuend, M., et al. (2018a). Cortical circuit activity evokes rapid astrocyte calcium signals on a similar timescale to neurons. Neuron 98, 726.e4-735.e4. doi: 10.1016/j. neuron.2018.03.050

Stobart, J. L., Ferrari, K. D., Barrett, M. J. P., Stobart, M. J., Looser, Z. J., Saab, A. S., et al. (2018b). Long-term in vivo calcium imaging of astrocytes reveals distinct cellular compartment responses to sensory stimulation. Cereb. Cortex 28, 184-198. doi: 10.1093/cercor/bhw366

Takata, N., and Hirase, H. (2008). Cortical layer 1 and layer 2/3 astrocytes exhibit distinct calcium dynamics in vivo. PLoS One 3:e2525. doi: 10.1371/journal. pone. 0002525

Takata, N., Mishima, T., Hisatsune, C., Nagai, T., Ebisui, E., Mikoshiba, K., et al. (2011). Astrocyte calcium signaling transforms cholinergic modulation to cortical plasticity in vivo. J. Neurosci. 31, 18155-18165. doi: 10.1523/JNEUROSCI.5289-11.2011

Tan, G., Sun, S.-Q., and Yuan, D.-L. (2008). Expression of Kir 4.1 in human astrocytic tumors: correlation with pathologic grade. Biochem. Biophys. Res. Commun. 367, 743-747. doi: 10.1016/j.bbrc.2008.01.014

Tang, X., Taniguchi, K., and Kofuji, P. (2009). Heterogeneity of Kir4.1 channel expression in glia revealed by mouse transgenesis. Glia 57, 1706-1715. doi: 10.1002/glia.20882

Verkhratsky, A. (2006). Glial calcium signaling in physiology and pathophysiology 1. Acta Pharmacol. Sin. 27, 773-780. doi: 10.1111/j.1745-7254.2006. 00396.x

Verkhratsky, A., and Nedergaard, M. (2018). Physiology of astroglia. Physiol. Rev. 98, 239-389. doi: 10.1152/physrev.00042.2016

Verkhratsky, A., Parpura, V., Vardjan, N., and Zorec, R. (2019). Physiology of astroglia. Adv. Exp. Med. Biol. 1175, 45-91. doi: 10.1007/978-981-13-99 13-8_3

Walker, M. C., and Kullmann, D. M. (2020). Optogenetic and chemogenetic therapies for epilepsy. Neuropharmacology 168:107751. doi: 10.1016/j. neuropharm.2019.107751

Walz, W. (1982). Do neuronal signals regulate potassium flow in glial cells? Evidence from an invertebrate central nenrous system. J. Neurosci. Res. 7, 71-79. doi: 10.1002/jnr.490070108

Wang, X., Lou, N., Xu, Q., Tian, G.-F., Peng, W. G., Han, X., et al. (2006) Astrocytic $\mathrm{Ca}^{2+}$ signaling evoked by sensory stimulation in vivo. Nat. Neurosci. 9, 816-823. doi: 10.1038/nn1703

Wang, F., Smith, N. A., Xu, Q., Fujita, T., Baba, A., Matsuda, T., et al. (2012). Astrocytes modulate neural network activity by $\mathrm{Ca}^{2+}$-dependent uptake of extracellular $\mathrm{K}^{+}$. Sci. Signal. 5:ra26. doi: 10.1126/scisignal.20 02334

Wang, M., Xu, Z., Liu, Q., Sun, W., Jiang, B., Yang, K., et al. (2019). Nongenetic optical modulation of neural stem cell proliferation and neuronal/glial differentiation. Biomaterials 225:119539. doi: 10.1016/j.biomaterials.2019. 119539

Wilhelm, I., Nyúl-Tóth, Á., Suciu, M., Hermenean, A., and Krizbai, I. A. (2016). Heterogeneity of the blood-brain barrier. Tissue Barriers 4:e1143544. doi: 10.1080/21688370.2016.1143544

Wilson, C. S., and Mongin, A. A. (2019). The signaling role for chloride in the bidirectional communication between neurons and astrocytes. Neurosci. Lett. 689, 33-44. doi: 10.1016/j.neulet.2018.01.012

Wu, Y.-W., Gordleeva, S., Tang, X., Shih, P.-Y., Dembitskaya, Y., Semyanov, A., et al. (2019). Morphological profile determines the frequency of spontaneous calcium events in astrocytic processes. Glia 67, 246-262. doi: 10.1002/glia. 23537 
Wu, K.-C., Kuo, C.-S., Chao, C.-C., Huang, C.-C., Tu, Y.-K., Chan, P., et al. (2015). Role of voltage-gated $\mathrm{K}^{+}$channels in regulating $\mathrm{Ca}^{2+}$ entry in rat cortical astrocytes. J. Physiol. Sci. 65, 171-177. doi: 10.1007/s12576-015-0356-9

Xin, W., Schuebel, K. E., Jair, K.-W., Cimbro, R., De Biase, L. M., Goldman, D., et al. (2019). Ventral midbrain astrocytes display unique physiological features and sensitivity to dopamine D2 receptor signaling. Neuropsychopharmacology 44, 344-355. doi: 10.1038/s41386-018-0151-4

Yu, X., Taylor, A. M. W., Nagai, J., Golshani, P., Evans, C. J., Coppola, G., et al. (2018). Reducing astrocyte calcium signaling in vivo alters striatal microcircuits and causes repetitive behavior. Neuron 99, 1170.e9-1187.e9. doi: 10.1016/j. neuron.2018.08.015

Zaitzev, A. V., Povysheva, N. V., Gonzalez-Burgos, G., and Lewis, D. A. (2012). Electrophysiological classes of layer $2 / 3$ pyramidal cells in monkey prefrontal cortex. J. Neurophysiol. 108, 595-609. doi: 10.1152/jn.00859.2011

Zhang, Y., Sloan, S. A., Clarke, L. E., Grant, G. A., Gephart, M. G. H., and Correspondence, B. A. B. (2016). Purification and characterization of progenitor and mature human astrocytes reveals transcriptional and functional differences with mouse. Neuron 89, 37-53. doi: 10.1016/j.neuron.2015. 11.013

Zhong, S., Du, Y., Kiyoshi, C. M., Ma, B., Alford, C. C., Wang, Q., et al. (2016). Electrophysiological behavior of neonatal astrocytes in hippocampal stratum radiatum. Mol. Brain 9:34. doi: 10.1186/s13041-016-0213-7

Zhou, M., Schools, G. P., and Kimelberg, H. K. (2006). Development of GLAST ${ }^{+}$ astrocytes and $\mathrm{NG}^{+}$glia in rat hippocampus CA1: mature astrocytes are electrophysiologically passive. J. Neurophysiol. 95, 134-143. doi: 10.1152/jn. 00570.2005

Zhou, M., Xu, G., Xie, M., Zhang, X., Schools, G. P., Ma, L., et al. (2009). TWIK1 and TREK-1 are potassium channels contributing significantly to astrocyte passive conductance in rat hippocampal slices. J. Neurosci. 29, 8551-8564. doi: 10.1523/JNEUROSCI.5784-08.2009

Zhu, J., Yan, J., and Thornhill, W. B. (2014). The Kv1.3 potassium channel is localized to the cis-Golgi and Kv1.6 is localized to the endoplasmic reticulum in rat astrocytes. FEBS J. 281, 3433-3445. doi: 10.1111/febs.12871

Zhu, H., Zhao, Y., Wu, H., Jiang, N., Wang, Z., Lin, W., et al. (2016). Remarkable alterations of Nav1.6 in reactive astrogliosis during epileptogenesis. Sci. Rep. 6:38108. doi: 10.1038/srep38108

Ziemens, D., Oschmann, F., Gerkau, N. J., and Rose, C. R. (2019). Heterogeneity of activity-induced sodium transients between astrocytes of the mouse hippocampus and neocortex: mechanisms and consequences. J. Neurosci. 39, 2620-2634. doi: 10.1523/JNEUROSCI.2029-18.2019

Zur Nieden, R., and Deitmer, J. W. (2006). The role of metabotropic glutamate receptors for the generation of calcium oscillations in rat hippocampal astrocytes in situ. Cereb. Cortex 16, 676-687. doi: 10.1093/cercor/ bhj013

Conflict of Interest: The authors declare that the research was conducted in the absence of any commercial or financial relationships that could be construed as a potential conflict of interest.

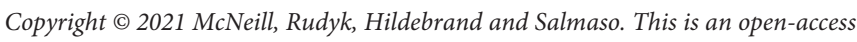
article distributed under the terms of the Creative Commons Attribution License (CC BY). The use, distribution or reproduction in other forums is permitted, provided the original author(s) and the copyright owner(s) are credited and that the original publication in this journal is cited, in accordance with accepted academic practice. No use, distribution or reproduction is permitted which does not comply with these terms. 